\title{
Optimum Dietary Ca/P Ratio and Phytase for Growth and Bone Mineralization in Juvenile Clarias gariepinus Fed Soya Bean-Based Diet
}

\author{
Akpoilih BU ${ }^{1 *}$, Ajani $\mathrm{EK}^{2}$ and Omitoyin $\mathrm{BO}^{2}$ \\ ${ }^{1}$ Department of Fisheries, University of Port Harcourt, Nigeria \\ ${ }^{2}$ Department of Aquaculture and Fisheries Management, University of Ibadan, Nigeria
}

\begin{abstract}
The research investigated the interactive effect of five phytase graded levels of $0 \mathrm{FTU} / \mathrm{g}(\mathrm{P0}), 250 \mathrm{FTU} / \mathrm{g}$ (P1), $500 \mathrm{FTU} / \mathrm{g}(\mathrm{P} 2), 750 \mathrm{FTU} / \mathrm{g}(\mathrm{P} 3), 1000 \mathrm{FTU} / \mathrm{g}$ (P4) and four graded levels of roasted soya bean substitution at $25 \%$, $50 \%, 75 \%$ and $100 \%$ in place of fish meal, respectively. All basal diets contained $0.20 \%$ calcium without added inorganic phosphorus, wheat and amino acids supplements so that the phosphorus, calcium, and $\mathrm{Ca} / \mathrm{P}$ ratios of the five (5) were: S0 (1.31\%, 1.85\%, and 1.41), S1 (1.15\%, 1.64\%, and 1.43), S2 (1.01\%, $1.28 \%$, and 1.27$)$, S3 $(0.75 \%$, $0.99 \%$, and 1.30$)$, and $\mathrm{S} 4(0.43 \%, 0.68 \%$, and 1.59$)$. 1092 fish of average weight $11.55 \pm 0.2 \mathrm{~g}$ were randomly allocated to all experimental diet and fed $3 \%$ body for 84 days. There was no significant difference in final weight, weight gain and FCR S3P1 and SOP0 ( $>>0.05)$. Fish fed low phosphorus diet ( $100 \%$ soya bean) showed reduced total weight and weight gain compared to high $(0 \%, 25 \%, 50 \%)$ and medium phosphorus diet $(75 \%)$ with phytase addition $(\mathrm{P}<0.05)$. Fish fed phytase at 50-200 FTU/g and inorganic control had lower weight gain compared to S3P1 $(P<0.05)$. Ca/P ratio correlated negatively with final weight $(r=-0.439, P<0.01)$, weight gain $(r=-0.431, P<0.01)$, SGR $(r=-0.138, P>0.05)$, and PER $(r=-0.210, P>0.05)$. Ca/P of S3P1 $(1.41 \pm 0.02)$ was not different from S0P0 $(1.41 \pm$ 0.01 ). Bone phosphorus, calcium and zinc were higher in $250 \mathrm{FTU} / \mathrm{g}$ compared to $0 \mathrm{FTU} / \mathrm{g}, 500 \mathrm{FTU} / \mathrm{g}, 750 \mathrm{FTU} / \mathrm{g}$ and $1000 \mathrm{FTU} / \mathrm{g}$. In conclusion, Phytase improved growth performance $(\mathrm{r}=0.05)$ of juvenile Clarias gariepinus with optimum at $250 \mathrm{FTU} / \mathrm{g}$; however, Ca/P affected performance $(\mathrm{r}=-0.431)$ and should range between 1.41-1.46 for optimum phytase activity.
\end{abstract}

Keywords: Phytase; Growth performance; Bone mineralization; $\mathrm{Ca} / \mathrm{P}$ ratio; Optimum

\section{Introduction}

The main source of plant protein in terrestrial and aquatic feed includes soybean meal, corn (gluten), groundnut meal, sunflower meal, canola/rapeseed meal, peas and lupins [1]. Soybean meal represents the highest proportion of plant protein in fish diets owing to high yield, relatively high crude protein content and easy and round the year availability. Soybeans are the most plentiful of oilseed crops, with a worldwide production of 253.1 million metric tonnes in 2012 [2]. However, the major constraints that limit use of plant proteins in animal feed are the presence of anti-nutritional factors such as saponins [3], lectins (Santiago et al.), and phytate being one of importance [1]. They are also limiting in essential amino acids affecting growth and nutrient utilization in fish [4]. Apart from minerals, phytate also forms complexes with proteins and amino acids [5] at acidic $\mathrm{pH}$ leading to the formation of phytate-protein aggregates and precipitates, which results in a decreased accessibility for proteases, and consequently in inefficient protein and amino acid digestion [6]. Cao et al. reported that the growth and feed conversion efficiency in commonly cultured fish species, such as carps, tilapias, trout and salmons were negatively affected by the phytate in the diets [7]. Phytase in fish diet can breakdown a portion of the phytate, increasing availability of dietary phytate-phosphorus in diets for fish $[1,8,9]$. However, very low phytase activities have been reported for legume seeds, hence dietary supplementation is necessary in oil seed meals, particularly soy bean meal to improve protein and mineral digestibility and availability, which is very low compared to fish meal [10]. Although dietary phytase supplementation results in substantial reductions in total phosphorus excretion, a slight increase of soluble phosphorus in the excreta is possible [11]. A precise prescription of appropriate phytase inclusion in diets is needed to release the actually required phosphorus and other nutrients for the animals to avoid or minimize this side effect [12], hence there is need to investigate the intrinsic (native) phytase in fish diets and the optimum phytase supplementation needed to promote growth and nutrient utilization in fish. In dose-response studies, phytase addition of 250-1500 FTU/g is usually considered feasible in many fish species. The optimum dose changes along with many factors such as fish species, different phytase sources, diet formulation (amount of substrate for phytase) and selected response parameters [7]. Optimum phytase has been determined for Channel catfish [13,14], Nile tilapia [15], Korean rockfish [16], Pangasius pangasius [17], and Rainbow trout [18]. Van weerd et al. [19] and Nwanna et al. [20] reported 750 FTU/g (Natuphos 5000) and $8000 \mathrm{FTU} / \mathrm{g}$ (Ronozyme 5000) phytase, respectively, as optimum for growth of Clarias gariepinus, but did not consider consistently increasing levels of soya bean used in their studies. In the former study, increasing levels of soya bean depressed growth performance, which could be due to the inclusion of wheat in the study. Wheat has a high phytase (1193 FTU/g), according to Eeckhout and de Paepe (1994) [47], which can compromise responses to phytase and reduce its efficacy.

In diet, it is necessary to take into account the $\mathrm{Ca} / \mathrm{P}$ ratio, because it has important consequences for bone development, which can be

*Corresponding author: Akpoilih BU, Department of Fisheries, University of Port Harcourt, Faculty of Agriculture, PMB 5323, Port Harcourt, Nigeria, Tel +2348134381645; E-mail: uzezi.u2@gmail.com

Received November 05, 2015; Accepted November 30, 2015; Published February 15, 2016

Citation: Akpoilih BU, Ajani EK, Omitoyin BO (2016) Optimum Dietary Ca/P Ratio and Phytase for Growth and Bone Mineralization in Juvenile Clarias gariepinus Fed Soya Bean-Based Diet. J Aquac Res Development 7: 403. doi:10.4172/21559546.1000403

Copyright: $\odot 2016$ Akpoilih BU, et al. This is an open-access article distributed under the terms of the Creative Commons Attribution License, which permits unrestricted use, distribution, and reproduction in any medium, provided the original author and source are credited. 
adversely affected when this ratio increases, resulting in aberrations in bone mineral homeostasis and bone metabolism [1]. Dietary levels of Ca:P ratios are crucial to phytase efficacy and activity [21]. Cao et al. [7] reported that data on this aspect in fish are limited. High dietary $\mathrm{Ca}$ or a high ratio of Ca: $\mathrm{P}$ interferes with phosphorus absorption and reduces the effectiveness of phytase activity $[22,23]$. High concentration of calcium in fish feed will chelate with phytate to become insoluble complex, or compete with phytase to change the phytase activity center site as an inhibitor, or increase the $\mathrm{pH}$ value to inhibit the activity of phytase. Increasing Ca:P ratio depressed $E$. coli derived phytase action in the pig diet and thus significantly depressed weight gain and feed efficiency. The aim of this research, therefore, is to define optimum dietary $\mathrm{Ca} / \mathrm{P}$ ratio and effective phytase efficacy for optimum growth performance in soya bean diet of juvenile Clarias gariepinus.

\section{Materials and Method}

\section{Experimental design}

The research investigated the interactive effect of five phytase supplemented at graded levels of $0 \mathrm{FTU} / \mathrm{g}$ (P0), $250 \mathrm{FTU} / \mathrm{g}$ (P1), 500 FTU/g (P2), 750 FTU/g (P3), 1000 FTU/g (P4) and substitution of four graded levels of fish meal with $25 \%, 50 \%, 75 \%$, and $100 \%$ roasted, fullfat soya bean meal with hulls as S1, S2, S3 and, S4 and fed to duplicate group of juvenile Clarias gariepinus in a $4 \times 5$ factorial design so that there are 21 treatment combinations with treatment code SOP0-S4P4 (S for soya bean, P for phytase). A diet with $100 \%$ fish meal (S0), which served as control, was included in the experiment, but with no phytase addition (P0).

\section{Experimental diet}

Five (5) iso-nitrogenous (40\%) and iso-caloric ( 18.15kj/g GE) diets serving as controls without dietary phytase were formulated using soya bean as the main plant protein source to replace fish meal at $0 \%$, $25 \%$ soya bean meal, $50 \%$ soya bean meal, $75 \%$ soya bean meal, and $100 \%$ soya bean meal, which were labelled as S0, S1, S2, S3 and S4 as basal diets served as controls with no phytase treatment (P0). Another four diets as commercial control was also included in this experimental group to compare growth performance with phytase supplementation in high (S1 and S2), medium (S3), and low phosphorus diet (S4) at 250 FTU/g (P1), 500 FTU/g (P2), 750 FTU/g (P3), and 1000 FTU/g (P4). A fish meal control (S0) was not supplemented with phytase (P0). All basal diets contained $0.20 \%$ calcium without added inorganic phosphorus, wheat and amino acids supplemented so that the phosphorus, calcium, and $\mathrm{Ca} / \mathrm{P}$ ratios of the five (5) were: $\mathrm{S} 0(1.31 \%, 1.85 \%$, and 1.41$), \mathrm{S} 1$ $(1.15 \%, 1.64 \%$, and 1.43$), \mathrm{S} 2(1.01 \%, 1.28 \%$, and 1.27$), \mathrm{S} 3(0.75 \%$, $0.99 \%$, and 1.30$)$, and $S 4(0.43 \%, 0.68 \%$, and 1.59$)$. Added calcium was lower than requirement of $0.45 \%$ for catfish [24] and 0.50 for African catfish (ADCP, 1983). Inorganic phosphorus was not added because phosphorus in feed, particularly at high levels can repress the activity of phytase. In addition, several studies have reported better growth performance with phytase compared to inorganic phosphorus source $[12,14]$. Moreover, Dicalcium phosphate has also been shown to lower phytase efficacy in poultry [25].

About $10 \mathrm{~kg}$ of soya bean was purchased from a local grain market with good quality soya bean products. Soybean was subjected to heat treatment by roasting [20] to remove anti-nutritional factors affecting nutrient bioavailability in fish; roasted soybean were grinded, sundried, and packed into plastic bags, and stored at ambient temperature prior to inclusion with other feeding stuff for the fish. Compounded feeds were formulated using Pearson's method and mixed with cold, distilled water to a moisture content of $14 \%$. Feed were cold pelleted and forced through a $2 \mathrm{~mm}$ hand mesh and sundried before phytase supplementation. Liquid Natuphos phytase (Natuphos 5000L, BASF, the Chemical Co., Germany) were prepared by serial dilution of the phytase concentrations using a dilution factor of 1:10 and bulk density of $1.2 \mathrm{~g} / \mathrm{cm}^{3}\left(1.15-1.25 \mathrm{~g} / \mathrm{cm}^{3}\right)$ according to BASF to give $0.4917 \mathrm{ml}, 0.9833 \mathrm{ml}, 1.475 \mathrm{ml}$, and $1.967 \mathrm{ml} / \mathrm{kg}$ diets for $250 \mathrm{FTU} / \mathrm{g}$, $500 \mathrm{FTU} / \mathrm{g}, 750 \mathrm{FTU} / \mathrm{g}$, and $1000 \mathrm{FTU} / \mathrm{g}$ phytase, respectively, which were supplemented in the diets according to the design. Phytase was supplied by BASF, the Chemical Co., Ludwigshafen, Germany, while phytase activity of all experimental groups analysed by BASF SE, Lampertheim, Germany. Both phytase and non phytase-supplemented groups were analysed for total phosphorus, phytate phosphorus [26], calcium, and phytase activity. The control diet, S0 (100\% fish meal), did not receive any phytase treatment. The gross and chemical composition of the basal diet is shown in Table 1 below.

\section{Experimental fish}

To meet the requirement of the $4 \times 5$ factorial design in the first experimental group pf roasted, full fat soya bean diets, about 1160 Clarias gariepinus fish of average weight $(4.5+0.2 \mathrm{~g})$, were procured from a reliable fish farm with no trace of metal contamination and pollution. Fish were acclimated under laboratory conditions for 3 weeks prior to experimental feeding during which time they were fed commercial diet in 10 fish holding tanks supplied with clean water until they attained the juvenile status of average weight $11.55+0.2 \mathrm{~g}$ and length $11.79+1.03$ $\mathrm{cm}$ before experimental feeding with phytase-treated diets. Water temperature, $\mathrm{pH}$ and oxygen maintained at optimum range between $25^{\circ} \mathrm{C}-32^{\circ} \mathrm{C}, 7.40-7.45,4.80-5.0 \mathrm{mg} / \mathrm{l}$. after which all fish were left unfed for 2-3 days prior to start of experiment. After acclimation, 1092 fish were randomly allocated to all 21 treatment combination groups of diets, including control diet, and fed to duplicate groups of fish stocked at 26 fish per tank 300.30g. Each of the feed rations were shared so that experimental fish were fed twice daily (morning at 08:00 hrs) and evening (evening at 16:00 hrs) to obtain optimum growth with uneaten feed and faeces removed at each feeding period. Experimental fish were weighed biweekly throughout the experiments using an electronic compact balance S. Mettler scale with accuracy of $0.01 \mathrm{~g}$ (Model: $\mathrm{K}-\mathrm{BH}$ ). Water was changed once every two (2) days using static water renewal method and water quality monitored and measured for all treatment tanks at the end of the experiment. Fish $(n=2)$ were sampled for bone mineralization after ashing to constant weight [27].

\section{Determination of mineral composition}

Duplicate sample of feeds were analyzed for phosphorus, calcium, sodium, copper, and manganese and determined using flame atomic absorption spectrophotometer model Buck 205, Buck Scientific, USA, while phosphorus $(\mathrm{P})$ was estimated spectrophotometrically using molybdovanadate method [28].

\section{Determination of phytate}

Phytate in feeds was measured by alkaline picrate method of Oberleas [23]. Sample was extracted with $0.2 \mathrm{~N} \mathrm{HCl}$ to give (3-30 $\mu \mathrm{g}$ $\mathrm{ml}^{-1}$ phytate solution). $0.5 \mathrm{ml}$ of extract was pipetted into a test tube fitted with a ground glass stopper. $1 \mathrm{ml}$ of ferric solution was added to the tube, which was covered with the stopper and fixed with a clip. The tube was heated in a boiling water bath for 30 minutes. Care was taken to ensure that for the first 5 minutes, the tube remained well stoppered. After cooling in ice water for 15 minutes, the tube was allowed to adjust to room temperature. Once the tube reached room temperature, the 


\begin{tabular}{|c|c|c|c|c|c|c|}
\hline Ingredient & $\mathrm{S}_{0}(0 \%)$ & $\mathrm{S}_{1}(25 \%)$ & $\mathrm{S}_{2}(50 \%)$ & $\mathrm{S}_{3}(75 \%)$ & $\mathrm{S}_{4}(100 \%)$ & $\mathrm{S}_{3} \mathrm{D}$ \\
\hline Fish meal $(66.46 \%)$ & 54.29 & 45.38 & 34.16 & 19.62 & - & 19.83 \\
\hline Soyabean meal (full fat, $42.93 \%$ ) & - & 15.13 & 34.16 & 58.85 & 92.15 & 59.48 \\
\hline Maize (10.24\%) & 41.71 & 35.50 & 27.68 & 17.54 & 3.87 & 16.69 \\
\hline Vit. Min Mix & 0.50 & 0.50 & 0.50 & 0.50 & 0.50 & 0.50 \\
\hline Fish oil & 1.00 & 1.00 & 1.00 & 1.00 & 1.00 & 1.00 \\
\hline $\mathrm{CaCO}_{3}$ & 0.50 & 0.50 & 0.50 & 0.50 & 0.50 & 0.50 \\
\hline Salt & 0.50 & 0.50 & 0.50 & 0.50 & 0.50 & 0.50 \\
\hline Chromic oxide & 0.50 & 0.50 & 0.50 & 0.50 & 0.50 & 0.50 \\
\hline Cellulose* & 0.20 & 0.20 & 0.20 & 0.20 & 0.20 & 0.20 \\
\hline Starch & 0.80 & 0.80 & 0.80 & 0.80 & 0.80 & 0.80 \\
\hline \multicolumn{7}{|l|}{ Chemical composition (\%) } \\
\hline Phosphorus & 1.31 & 1.15 & 1.01 & 0.75 & 0.43 & 1.23 \\
\hline Available phosphorus & 0.91 & 0.63 & 0.44 & 0.33 & 0.03 & \\
\hline Calcium & 1.85 & 1.64 & 1.28 & 0.99 & 0.68 & 3.63 \\
\hline Magnesium & 0.25 & 0.24 & 0.28 & 0.27 & 0.27 & 0.16 \\
\hline Potassium & 0.87 & 1.10 & 1.35 & 1.60 & 1.90 & 1.53 \\
\hline Zinc & 15.82 & 14.22 & 12.59 & 12.12 & 12.40 & 17.81 \\
\hline $\mathrm{Ca} / \mathrm{P}$ ratio & 1.41 & 1.43 & 1.27 & 1.30 & 1.59 & 2.95 \\
\hline Phytate & 0.40 & 0.52 & 0.57 & 0.43 & 0.40 & \\
\hline Native phytase (FTU/g) & $<100$ & $<100$ & $<100$ & $<100$ & $<100$ & $<100$ \\
\hline
\end{tabular}

\#Micro mineral mix contains per kilogram: Vit. A (20, 000 IU), Vit. D3 (5, 000 IU), Vit. E (300 mg), Vit K3 (10 mg), Vit. B1 (20 mg), Vit. B2 (25 mg), Vit. C (300 mg), Niacin $(120 \mathrm{mg})$, Ca Pantothenate $(60 \mathrm{mg})$, Vit. B6 (10 mg), Vit. B12 (0.05), Folic acid (5 mg), Biotin (1 mg), Choline chloride (5 mg), Inositol (50 mg), Manganese (30 mg), Iron (35 mg), Zincn (45 mg), Copper (3 mg), lodine (5 mg), Cobalt (2 mg), Lysine (85 mg), Selenium (0.15 mg), Antooxidant (80 mg), Methionine (100 mg).

* Ascarboxymethyl cellulose

content of the tube was mixed and centrifuged for 30 minutes at 3000 g. $1 \mathrm{ml}$ of the supernatant was transferred to another test tube and 1.5 $\mathrm{ml}$ of 2, 2'-Bipyridine solution added. The absorbance was measured at $519 \mathrm{~nm}$ against distilled water.

\section{Determination of phytase activity}

"Phytase activity in feed sample was determined by Engelen et al. [30] with slight modifications according to the relative method of BASF [29] using methods by Engelen et al. [30]. $100 \mathrm{~g}$ of each sample were incubated with sodium phytate as substrate, which was dissolved with acetic buffer. The incubation was stopped by adding acidic molybdate/ vanadate reagent to produce a colored complex with the phosphate formed. The enzyme phytase liberates inorganic phosphate from the substrate sodium phytate during incubation and the intensity of the yellow color of the vanadomolybdophosphorus complex is a measure of the amount of phosphate liberated and was measured at a wavelength of $415 \mathrm{~nm}$.

\section{Growth and nutrient utilization}

Growth performance was monitored biweekly with the following parameters measured:

Weight gain=Final weight-initial weight

Feed Conversion Ratio (FCR): Feed Intake (g)/Fish weight gain (g)

Specific Growth Rate (\%): In (W2-W2) / (t2-t1), where W2 and W1 are weights on day $\mathrm{t} 2$ and $\mathrm{t} 1$, respectively.

Where, $\mathrm{W} 2=$ final weight, $\mathrm{W} 1=$ =initial weight, $\mathrm{t} 2=$ time at the end of experiment, $\mathrm{t} l=$ time at end of experiment

Survival rate $(\%)=$ Initial number of fish-Mortality/Initial number of fish $\times 100$

\section{Bone mineral analysis}

At the beginning and end of each feeding trial, bones of fish sacrificed for each experiment were investigated for calcium, phosphorus, magnesium, iron and zinc analysis described as follows:

Intact spinal, skull, pectoral, dorsal and caudal bones were removed from each group of fish and pooled for bone mineral analysis. Whole fish flesh was stripped off from the vertebrae (spinal) and the adhering flesh removed by light brushing and rinsing in distilled water. The vertebrae were oven-dried dried for 2 hours at $110^{\circ} \mathrm{C}$ and then pulverized, dried again, and weighed. The dried samples were ashed at $550^{\circ} \mathrm{C}$ for $6 \mathrm{~h}$. Ash weight was calculated as a percentage of dry, fat-free bone weight. For mineral estimation, the ash was digested in a boiling nitric acid and perchloric acid mixture (2:1) according to the method by AOAC [28]. After appropriate dilution, calcium, magnesium, potassium, zinc content was estimated by atomic absorption spectrophotometer using flame atomic absorption spectrophotometer model Buck 205, Buck Scientific, USA, while phosphorus was estimated spectrophotometrically using molybdovanadate method [28].

\section{Determination of phosphorus retention efficiency}

Phosphorus retention efficiency was determined by method of Cao et al. [31] as,

Phosphorus retention efficiency=Phosphorus retained/Phosphorus fed

Phosphorus retained $=\left(\mathrm{FBW} \times \mathrm{F}_{\mathrm{p}}\right)-\left(\mathrm{IBW} \times \mathrm{I}_{\mathrm{p}}\right)$

Phosphorus fed=Dp $\times$ FI

Where, FBW=Final Body Weight; IBW=Initial Body Weight; 
$\mathrm{F}_{\mathrm{p}}=$ Phosphorus in final carcass; $\mathrm{I}_{\mathrm{p}}=$ Phosphorus in initial carcass; $\mathrm{D}_{\mathrm{p}}=$ Phosphorus in diet

\section{Statistical analysis}

All data were subjected to one-way analysis of variance at significance level of 0.05 . Individual differences in treatment means were determined by Duncan new multiple range test [32]. Interactions between dietary levels of phytase and experimental diets were determined using multiple regression analysis (factorial). Data was treated as mean+S.D. A dose-response data or growth model [33] of graded phytase inclusion was determined by the linear equation below.

$$
\mathrm{Y}=\mathrm{a}_{0}+\mathrm{a}_{1} \mathrm{X}
$$

\section{Results}

\section{Mineral composition of diet}

With the exception of SOP0 diet (fish meal based), there was a highly significant improvement in phosphorus levels from $1.15 \pm$ $0.01 \%$ in S1P0 to $1.21 \pm 0.01 \%$ in S1P4. Diet S2P4 showed a significant increase $(\mathrm{P}<0.05)$ in phosphorus $(0.86 \pm 0.01 \%)$ compared to S2P3 $(0.76 \pm 0.01 \%), \mathrm{S} 2 \mathrm{P} 2(0.83 \pm 0.01 \%)$. There were no significant phytase effects $(\mathrm{P}<0.05)$ between diets S4P4, S4P0, and S4P1; S4P0, S4PI, S4P3; and between diets S3P4, S3P1, and S3P0, although phosphorus level for S3P1 $(0.76 \pm 0.01 \%)$ was higher than diets S3P0 $(0.75 \pm 0.01 \%)$ and S3P4 $(0.75 \pm 0.01 \%)(\mathrm{P}>0.05)$. Diets with $100 \%$ soya bean had the lowest values of phosphorus, which ranged between $(0.40 \pm 0.02 \%)$ in S4P2 to $0.45 \pm 0.01 \%$ in $54 \mathrm{P} 3$. Phytase significantly $(\mathrm{P}<0.05)$ affected available phosphorus with significant increase from S1P0 $(0.63 \pm 0.01 \%)$ to S1P4 $(1.03 \pm 0.02 \%)$, which was not significantly different $(\mathrm{P}>0.05)$ from S1P1 (1.03 $\pm 0.02 \%)$, but higher than S0P0 (0.91 $\pm 0.01 \%)$. S1P4 recorded highest available level, while S4P3 showed the lowest $(0.01 \pm$ $0.01 \%)$. Diet S3P1 $(0.42 \pm 0.02 \%)$ had the highest available phosphorus level compared to S3P0 $(0.33 \pm 0.01 \%)$, S3P2 $(0.21 \pm 0.01 \%)$, S3P3 $(0.24 \pm 0.01 \%)$, and S3P4 $(0.31 \pm 0.02 \%)$, which was not significantly different from S3P0 $(\mathrm{P}>0.05)$. There were no also differences between diets S4P1 $(0.23 \pm 0.01 \%)$ and S4P4 $(0.16 \pm 0.01 \%)$; and between S3P2, S4P1, S3P3 $(\mathrm{P}>0.05)$. Phytase supplementation significantly improved calcium levels in all soyabean diets compared to control diets, except S0P0, without phytase $(\mathrm{P}<0.05)$. Diets S1P1 $(1.70 \pm 0.01 \%)$ and S1P3 $(1.70 \pm 0.01 \%)$, which were higher than S1P0 $(1.64 \pm 0.01 \%)$, S1P2 $(1.59 \pm 0.01 \%)$, and S1P4 $(1.56 \pm 0.01 \%)(\mathrm{P}<0.05)$. Phytase improved significantly calcium levels from $0.99 \pm 0.01 \%$ (S3P0) to $1.20 \pm 0.01 \%$ (S3P4) $(\mathrm{P}<0.05)$. There was no significant difference between S4P4 $(0.72 \pm 0.01 \%)$ and S4P3 $(0.72 \pm 0.01 \%)(\mathrm{P}>0.05)$. Calcium levels was highest in S0P0 $(1.85 \pm 0.01 \%)$ and lowest in S4P3 $(0.72 \pm 0.01 \%)$ Phytase supplementation increased significantly zinc levels in diets with $25 \%, 50 \%$, and slightly in $75 \%$ soya bean meal (S3P1). Values increased from $14.22 \pm 0.01 \%(\mathrm{~S} 1 \mathrm{P} 0)$ to $15.01 \pm 0.01 \%$ (S1P4). There was a significant increase in zinc levels from $12.59 \pm 0.02 \%$ (S2P0) to $12.89 \pm 0.01 \%$ (S2P4). Dietary supplementation in $75 \%$ soya bean showed a statistically significant increase $(\mathrm{P}<0.05)$ in zinc levels for S3P1 (12.48 $\pm 0.01 \%)$ compared to S3P0 (12.12 $\pm 0.02 \%)$, S3P2 (12.19 $\pm 0.01 \%), \mathrm{S} 3 \mathrm{P} 3(11.85 \pm 0.01 \%)$, and S3P4 $(12.30 \pm 0.01 \%)$. Dietary copper was significantly improved with phytase addition to all soya bean diet $(\mathrm{P}<0.05)$. Mineral composition of diet based on full fat soyabean is shown in Table 2.

\section{Growth and nutrient utilization}

Final weight (Table 3) ranged from $373.84 \pm 43.78 \mathrm{~g}$ (S4P0) to $1526.56 \pm 64.78 \mathrm{~g}$ (S0P0). There were, however, no statistically significant effects $(\mathrm{P}>0.05)$ of phytase on final weight for diets S0P0, S1P0, S1P1, S1P2, S1P3, and S1P4; S2P0, S2P1, and S2P2; S2P1, S2P2, S3P0, S3P2, and S3P3; S4P0, S4P1, S4P2, S4P3, and S4P4. Final weight of fish fed $250 \mathrm{FTU} / \mathrm{g}$ recorded the highest value compared with fish fed 0FTU/g, $500 \mathrm{FTU} / \mathrm{g}, 750 \mathrm{FTU} / \mathrm{g}$, and 1000FTU/g. Fish fed low phosphorus diet ( $100 \%$ soya bean) showed reduced total weight compared to high $(0 \%, 25 \%, 50 \%)$ and medium phosphorus diet. Phytase increased significantly total weight of fish fed $25 \%$ soya bean diets (S1) from

Table 2: Mineral composition of experimental diet based on full fat and supplemented with phytase.

\begin{tabular}{|c|c|c|c|c|c|c|}
\hline Treatments & Phosphorus (\%) & $\begin{array}{c}\text { Available } \\
\text { Phosphorus (\%) }\end{array}$ & Calcium (\%) & Magnesium (\%) & Potassium (\%) & Zinc (ppm) \\
\hline SOPO & $1.31 \pm 0.01^{m}$ & $0.91 \pm 0.01^{j}$ & $1.85 \pm 0.01^{q}$ & $0.25 \pm 0.01^{\mathrm{bc}}$ & $0.87 \pm 0.01^{a}$ & $15.82 \pm 0.02^{\mathrm{s}}$ \\
\hline S1P0 & $1.15 \pm 0.01^{k}$ & $0.63 \pm 0.01^{h}$ & $1.64 \pm 0.01^{n}$ & $0.24 \pm 0.02^{b}$ & $1.10 \pm 0.01^{c}$ & $14.22 \pm 0.01^{n}$ \\
\hline S1P1 & $1.17 \pm 0.01^{k}$ & $1.03 \pm 0.02^{k}$ & $1.70 \pm 0.01^{p}$ & $0.26 \pm 0.01^{\mathrm{bcd}}$ & $1.08 \pm 0.01^{b c}$ & $14.87 \pm 0.01^{q}$ \\
\hline S1P2 & $1.21 \pm 0.01^{\prime}$ & $0.60 \pm 0.01^{\mathrm{h}}$ & $1.59 \pm 0.01^{m}$ & $0.27 \pm 0.01^{\text {cde }}$ & $1.09 \pm 0.01^{\mathrm{bc}}$ & $14.41 \pm 0.01^{\circ}$ \\
\hline S1P3 & $1.17 \pm 0.01^{\mathrm{k}}$ & $0.66 \pm 0.01^{i}$ & $1.70 \pm 0.01^{p}$ & $0.26 \pm 0.01^{\mathrm{bcd}}$ & $1.06 \pm 0.01^{b}$ & $14.70 \pm 0.01^{p}$ \\
\hline S1P4 & $1.21 \pm 0.01^{\prime}$ & $1.03 \pm 0.02^{\mathrm{k}}$ & $1.56 \pm 0.01^{\prime}$ & $0.27 \pm 0.01^{\text {cde }}$ & $1.07 \pm 0.01^{b}$ & $15.01 \pm 0.01^{r}$ \\
\hline S2P0 & $1.01 \pm 0.01^{j}$ & $0.44 \pm 0.01^{\mathrm{fg}}$ & $1.28 \pm 0.01^{i}$ & $0.28 \pm 0.02^{\text {def }}$ & $1.35 \pm 0.01^{\mathrm{e}}$ & $12.59 \pm 0.02^{k}$ \\
\hline $\mathrm{S} 2 \mathrm{P} 1$ & $0.90 \pm 0.01^{i}$ & $0.46 \pm 0.01^{\mathrm{g}}$ & $1.35 \pm 0.01^{k}$ & $0.28 \pm 0.01^{\text {def }}$ & $1.34 \pm 0.01^{\mathrm{e}}$ & $12.79 \pm 0.01^{\prime}$ \\
\hline $\mathrm{S} 2 \mathrm{P} 2$ & $0.83 \pm 0.01^{g}$ & $0.43 \pm 0.02^{f}$ & $1.66 \pm 0.01^{\circ}$ & $0.36 \pm 0.01^{h}$ & $1.28 \pm 0.02^{d}$ & $12.44 \pm 0.01^{i}$ \\
\hline S2P3 & $0.76 \pm 0.01^{\mathrm{e}}$ & $0.37 \pm 0.01^{\mathrm{e}}$ & $1.36 \pm 0.01^{k}$ & $0.28 \pm 0.01^{\text {ef }}$ & $1.34 \pm 0.01^{\mathrm{e}}$ & $12.90 \pm 0.01^{m}$ \\
\hline $\mathrm{S} 2 \mathrm{P} 4$ & $0.86 \pm 0.01^{\mathrm{h}}$ & $0.68 \pm 0.01^{i}$ & $1.32 \pm 0.01^{j}$ & $0.28 \pm 0.01^{\text {def }}$ & $1.36 \pm 0.01^{\mathrm{e}}$ & $12.89 \pm 0.01^{\mathrm{m}}$ \\
\hline S3P0 & $0.75 \pm 0.01^{\mathrm{e}}$ & $0.33 \pm 0.01^{d}$ & $0.99 \pm 0.01^{\mathrm{e}}$ & $0.27 \pm 0.01^{\text {cde }}$ & $1.60 \pm 0.01^{\mathrm{h}}$ & $12.12 \pm 0.02^{\mathrm{e}}$ \\
\hline S3P1 & $0.76 \pm 0.01^{e}$ & $0.42 \pm 0.02^{f}$ & $1.06 \pm 0.01^{f}$ & $0.28 \pm 0.01^{\text {def }}$ & $1.60 \pm 0.01^{h}$ & $12.48 \pm 0.01^{j}$ \\
\hline S3P2 & $0.72 \pm 0.01^{d}$ & $0.21 \pm 0.01^{c}$ & $1.05 \pm 0.01^{\dagger}$ & $0.31 \pm 0.01^{\mathrm{g}}$ & $1.50 \pm 0.01^{f}$ & $12.19 \pm 0.01^{f}$ \\
\hline S3P3 & $0.78 \pm 0.01^{f}$ & $0.24 \pm 0.01^{c}$ & $1.17 \pm 0.01^{\mathrm{g}}$ & $0.29 \pm 0.01^{\mathrm{fg}}$ & $1.58 \pm 0.01^{h}$ & $11.85 \pm 0.01^{d}$ \\
\hline S3P4 & $0.75 \pm 0.01^{\mathrm{e}}$ & $0.31 \pm 0.02^{d}$ & $1.20 \pm 0.01^{\mathrm{h}}$ & $0.29 \pm 0.01^{\mathrm{efg}}$ & $1.56 \pm 0.01^{g}$ & $12.30 \pm 0.01^{9}$ \\
\hline S4P0 & $0.43 \pm 0.01^{b c}$ & $0.03 \pm 0.01^{a}$ & $0.68 \pm 0.01^{b}$ & $0.27 \pm 0.01^{\text {cde }}$ & $1.90 \pm 0.01^{j}$ & $12.40 \pm 0.01^{\mathrm{h}}$ \\
\hline S4P1 & $0.44 \pm 0.01^{\mathrm{bc}}$ & $0.23 \pm 0.01^{c}$ & $0.65 \pm 0.01^{a}$ & $0.28 \pm 0.01^{\text {def }}$ & $1.75 \pm 0.01^{i}$ & $11.82 \pm 0.01^{\circ}$ \\
\hline S4P2 & $0.40 \pm 0.02^{\mathrm{a}}$ & $0.15 \pm 0.01^{b}$ & $0.82 \pm 0.01^{\mathrm{d}}$ & $0.29 \pm 0.01^{\mathrm{efg}}$ & $1.96 \pm 0.01^{\prime}$ & $11.73 \pm 0.02^{a}$ \\
\hline S4P3 & $0.45 \pm 0.01^{c}$ & $0.01 \pm 0.01^{a}$ & $0.72 \pm 0.01^{c}$ & $0.29 \pm 0.01^{\mathrm{efg}}$ & $1.92 \pm 0.01^{k}$ & $12.49 \pm 0.01^{j}$ \\
\hline S4P4 & $0.42 \pm 0.01^{\mathrm{ab}}$ & $0.16 \pm 0.01^{b}$ & $0.72 \pm 0.01^{c}$ & $0.20 \pm 0.01^{a}$ & $1.96 \pm 0.01^{\prime}$ & $11.76 \pm 0.01^{b}$ \\
\hline
\end{tabular}

Mean values with the same alphabet superscript in the same column are not significantly different at the 0.05 level (2-tailed) 
Table 3: Growth parameters and nutrient utilization of Clarias gariepinus fed experimental diet (full fat).

\begin{tabular}{|c|c|c|c|c|c|c|c|c|}
\hline Trt & Initial weight & Final weight (g) & Weight gain (g) & SGR (\%) & FCR & Survival rate (\%) & $\mathrm{Ca} / \mathrm{P}$ ratio & $\begin{array}{l}\text { Analysed Phytase } \\
\text { Activity (FTU/g) }\end{array}$ \\
\hline SOPO & $309.09 \pm 14.38$ & $1526.56 \pm 64.78^{\mathrm{g}}$ & $1219.47 \pm 47.57^{f}$ & $1.90 \pm 0.01^{\mathrm{ef}}$ & $1.29 \pm 0.01^{\mathrm{a}}$ & $92.31 \pm 0.00^{\text {cd }}$ & $1.41 \pm 0.00^{b c}$ & $<100$ \\
\hline S1P0 & $312.99 \pm 0.06$ & $1351.26 \pm 7.55^{\mathrm{fg}}$ & $1043.78 \pm 0.29^{e f}$ & $1.74 \pm 0.01^{\text {cdef }}$ & $1.38 \pm 0.08^{a}$ & $100.00 \pm 0.00^{d}$ & $1.43 \pm 0.00^{\mathrm{bcd}}$ & $<100$ \\
\hline S1P1 & $330.47 \pm 8.74$ & $1413.18 \pm 102.09^{f g}$ & $1082.71 \pm 110.82^{\text {ef }}$ & $1.72 \pm 0.03^{\text {cdef }}$ & $1.39 \pm 0.06^{a}$ & $98.08 \pm 2.72^{\mathrm{d}}$ & $1.45 \pm 0.00^{\text {bcd }}$ & 580 \\
\hline S1P2 & $308.77 \pm 16.06$ & $1252.15 \pm 10.01^{\text {def }}$ & $943.39 \pm 26.07^{\mathrm{de}}$ & $1.43 \pm 0.11^{\text {cde }}$ & $1.57 \pm 0.22^{\mathrm{ab}}$ & $100.00 \pm 0.00^{d}$ & $1.33 \pm 0.01^{\text {as }}$ & 720 \\
\hline S1P3 & $370.97 \pm 17.65$ & $1433.75 \pm 110.61^{\text {fg }}$ & $1082.78 \pm 121.28^{\mathrm{ef}}$ & $1.67 \pm 0.09^{\text {cdef }}$ & $1.47 \pm 0.11^{a}$ & $96.16 \pm 5.44^{d}$ & $1.45 \pm 0.00^{\mathrm{bcd}}$ & 890 \\
\hline S1P4 & $294.81 \pm 52.13$ & $1337.42 \pm 3.80^{\mathrm{efg}}$ & $1042.62 \pm 48.33^{\text {ef }}$ & $1.73 \pm 0.12^{\text {cdef }}$ & $1.37 \pm 0.13^{a}$ & $98.08 \pm 2.72^{\mathrm{d}}$ & $1.29 \pm 0.02^{\mathrm{a}}$ & 1510 \\
\hline S2P0 & $304.78 \pm 13.85$ & $1290.05 \pm 30.28^{\text {def }}$ & $985.27 \pm 16.43^{\mathrm{de}}$ & $1.66 \pm 0.04^{\text {cdef }}$ & $1.34 \pm 0.08^{a}$ & $86.54 \pm 2.72^{c}$ & $1.27 \pm 0.02^{\mathrm{a}}$ & $<100$ \\
\hline S2P1 & $270.65 \pm 10.34$ & $1096.13 \pm 82.38^{\mathrm{cd}}$ & $825.49 \pm 72.03^{\text {cd }}$ & $1.66 \pm 0.03^{\text {cdef }}$ & $1.40 \pm 0.01^{\mathrm{a}}$ & $94.23 \pm 2.72^{\text {cd }}$ & $1.50 \pm 0.04^{\mathrm{de}}$ & 270 \\
\hline S2P2 & $284.98 \pm 25.05$ & $1133.55 \pm 110.37^{\text {cde }}$ & $848.57 \pm 85.32^{\mathrm{cd}}$ & $1.69 \pm 0.04^{\text {cdef }}$ & $1.38 \pm 0.01^{a}$ & $86.54 \pm 8.16^{c}$ & $2.00 \pm 0.02^{h}$ & 580 \\
\hline S2P3 & $314.79 \pm 35.94$ & $1428.10 \pm 244.04^{\mathrm{fg}}$ & $1113.31 \pm 208.10^{\mathrm{ef}}$ & $1.66 \pm 0.06^{\text {cdef }}$ & $1.29 \pm 0.08^{a}$ & $100.00 \pm 0.00^{d}$ & $1.80 \pm 0.03^{g}$ & 1260 \\
\hline $\mathrm{S} 2 \mathrm{P} 4$ & $294.05 \pm 19.85$ & $1277.64 \pm 23.77^{\text {def }}$ & $983.60 \pm 43.62^{\mathrm{de}}$ & $1.69 \pm 0.06^{\text {cdef }}$ & $1.34 \pm 0.18^{a}$ & $94.23 \pm 2.72^{\text {cd }}$ & $1.54 \pm 0.00^{\mathrm{ef}}$ & 1260 \\
\hline S3P0 & $312.41 \pm 13.59$ & $1040.13 \pm 142.81^{c}$ & $727.72 \pm 129.22^{c}$ & $1.39 \pm 0.22^{\mathrm{cd}}$ & $1.67 \pm 0.12^{\mathrm{ab}}$ & $98.08 \pm 2.72^{\mathrm{d}}$ & $1.30 \pm 0.00^{\mathrm{a}}$ & $<100$ \\
\hline S3P1 & $251.59 \pm 68.82$ & $1366.47 \pm 89.64^{\mathrm{fg}}$ & $1114.88 \pm 20.82^{\text {ef }}$ & $2.04 \pm 0.00^{9}$ & $1.30 \pm 0.06^{\mathrm{a}}$ & $73.08 \pm 10.88^{b}$ & $1.41 \pm 0.02^{\mathrm{b}}$ & 330 \\
\hline S3P2 & $342.73 \pm 25.84$ & $1093.83 \pm 110.35^{\mathrm{cd}}$ & $751.10 \pm 136.19^{c}$ & $1.67 \pm 0.13^{\text {cdef }}$ & $1.74 \pm 0.22^{\mathrm{ab}}$ & $96.15 \pm 0.00^{d}$ & $1.46 \pm 0.00^{\text {bcde }}$ & 660 \\
\hline S3P3 & $311.63 \pm 5.52$ & $999.23 \pm 92.2^{c}$ & $687.60 \pm 86.78^{c}$ & $1.80 \pm 0.06^{\text {cdef }}$ & $2.40 \pm 0.18^{b}$ & $94.23 \pm 2.72^{\mathrm{cd}}$ & $1.49 \pm 0.02^{\text {cde }}$ & 1050 \\
\hline S3P4 & $327.72 \pm 34.65$ & $992.13 \pm 15.21^{c}$ & $664.41 \pm 19.44^{c}$ & $1.38 \pm 0.10^{\mathrm{cd}}$ & $2.36 \pm 0.23^{b}$ & $94.23 \pm 2.72^{\mathrm{cd}}$ & $1.60 \pm 0.02^{f}$ & 1340 \\
\hline S4P0 & $310.44 \pm 32.31$ & $373.84 \pm 43.78^{a}$ & $63.41 \pm 11.48^{a}$ & $0.22 \pm 0.1^{a}$ & $16.71 \pm 1.43^{e}$ & $75.00 \pm 2.72^{b}$ & $1.59 \pm 0.04^{f}$ & $<100$ \\
\hline S4P1 & $317.25 \pm 41.10$ & $635.44 \pm 54.15^{\mathrm{ab}}$ & $318.19 \pm 13.05^{b}$ & $0.83 \pm 0.04^{b}$ & $3.30 \pm 0.37^{\circ}$ & $92.31 \pm 0.00^{\text {cd }}$ & $1.48 \pm 0.04^{\text {bcde }}$ & 330 \\
\hline S4P2 & $293.01 \pm 13.91$ & $488.40 \pm 22.60^{\mathrm{ab}}$ & $195.40 \pm 8.70^{\mathrm{ab}}$ & $0.61 \pm 0.00^{b}$ & $4.43 \pm 0.06^{d}$ & $96.16 \pm 5.44^{d}$ & $2.07 \pm 0.13^{h}$ & 640 \\
\hline S4P3 & $309.04 \pm 12.0$ & $526.81 \pm 60.99^{a b}$ & $217.77 \pm 48.99^{\mathrm{ab}}$ & $0.63 \pm 0.07^{b}$ & $4.49 \pm 0.54^{d}$ & $94.23 \pm 2.72^{\mathrm{cd}}$ & $1.61 \pm 0.01^{f}$ & 830 \\
\hline S4P4 & $312.77 \pm 22.65$ & $458.48 \pm 26.87^{\mathrm{ab}}$ & $145.56 \pm 49.30^{\mathrm{ab}}$ & $0.90 \pm 0.78^{b}$ & $4.97 \pm 0.48^{\mathrm{d}}$ & $55.19 \pm 1.90^{\mathrm{a}}$ & $1.73 \pm 0.00^{\mathrm{g}}$ & 930 \\
\hline Sig. & $>0.05$ & $<0.05$ & $<0.05$ & $<0.05$ & $<0.05$ & $<0.05$ & $<0.05$ & \\
\hline
\end{tabular}

Mean values with the same alphabet superscript in the same column are not significantly different at the 0.05 level (2-tailed)

Table 4: Effects of phytase on growth and nutrient utilization in Clarias gariepinus fed soyabean based diet.

\begin{tabular}{|c|c|c|c|c|c|c|c|}
\hline Phytase (FTU/g) & Initial weight & Final weight (g) & Weight gain (g) & SGR & FCR & Survival rate (\%) & Ca /P \\
\hline 0 & $309.94 \pm 13.80$ & $1116.37 \pm 428.04$ & $807.93 \pm 428.67$ & $1.46 \pm 0.48$ & $4.48 \pm 6.47$ & $90.38 \pm 9.64$ & $1.40 \pm 0.12^{\mathrm{a}}$ \\
\hline 250 & $292.49 \pm 46.32$ & $1127.8 \pm 336.33$ & $835.32 \pm 344.72$ & $1.55 \pm 0.60$ & $1.85 \pm 0.91$ & $89.42 \pm 11.21$ \\
\hline 500 & $307.37 \pm 28.45$ & $991.98 \pm 322.57$ & $684.61 \pm 316.63$ & $1.42 \pm 0.39$ & $2.28 \pm 1.34$ & $94.71 \pm 6.48$ \\
\hline 750 & $326.61 \pm 31.76$ & $1096.97 \pm 413.94$ & $775.36 \pm 400.46$ & $1.25 \pm 0.66$ & $2.41 \pm 1.38$ & $96.15 \pm 3.56$ & $1.59 \pm 0.144^{\mathrm{a}}$ \\
\hline 1000 & $307.33 \pm 30.20$ & $1016.42 \pm 371.84$ & $709.04 \pm 381.61$ & $1.42 \pm 0.46$ & $2.51 \pm 1.60$ & $85.43 \pm 18.84$ & $1.54 \pm 0.175^{\mathrm{b}}$ \\
\hline Total & $308.80 \pm 31.45$ & $1072.12 \pm 364.99$ & $764.62 \pm 365.09$ & $1.42 \pm 0.51$ & $2.79 \pm 3.37$ & $91.18 \pm 11.24$ \\
\hline
\end{tabular}

$1351.26 \pm 7.55 \mathrm{fg}(\mathrm{S} 1 \mathrm{P} 0)$ to $1433.75 \pm 110.61 \mathrm{~g}(\mathrm{~S} 1 \mathrm{P} 3)$. Fish fed S1P2 recorded the lowest $(943.39 \pm 26.07 \mathrm{~g})$. Fish fed S2P1 (825.49 \pm 72.03 g) had the lowest weight gain for diets based on $50 \%$ soya bean meal (S2) with phytase increasing weight gain from $985.27 \pm 16.43 \mathrm{~g}(\mathrm{~S} 2 \mathrm{P} 0)$ to $1113.31 \pm 208.10 \mathrm{~g}(\mathrm{~S} 2 \mathrm{P} 3)$. S2P4 fish was not significantly different from S2P1 fish in weight gain ( $\mathrm{P}>0.05)$. Phytase inclusion in $75 \%$ soya bean diet (S3) resulted in highest weight gain for S3P1 fish (1114.88 \pm $20.82 \mathrm{~g})$, which was statistically different from S3P2 $(751.10 \pm 136.19$ g), S3P3 $(687.60 \pm 86.78 \mathrm{~g})$, and S3P4 $(664.41 \pm 19.44 \mathrm{~g})(\mathrm{P}<0.05)$. There was, however, no significant difference between S3P1, S0P0, $\mathrm{S} 1 \mathrm{P} 0, \mathrm{~S} 1 \mathrm{P} 1, \mathrm{~S} 1 \mathrm{P} 3, \mathrm{~S} 1 \mathrm{P} 4$, and S2P3 fish $(\mathrm{P}>0.05)$. Weight gain increased significantly with phytase addition to diet based on $100 \%$ (S4) soya bean meal $(\mathrm{P}<0.05)$. Fish fed S4P4 had the lowest weight gain compared to S4P1, S4P2, and S4P3.Weight gain of fish fed $100 \%$ was, however, significantly lower compared to fish fed $0 \%$ (S0), 25\% (S1), 50\% (S2) and $75 \%$ (S3) soya bean meal (Table 4). Figure 1 showed weight gain by fish fed soya bean diet (full fat) supplemented with phytase with the lowest values recorded $100 \%$ soya bean. There was also a strong, but negative, relationship (correlation) between final weight of fish and calcium/phosphorus ratio (Table 5) in diet $(\mathrm{r}=-0.439, \mathrm{P}<0.05)$, while regression coefficient (Table 5 and Figures 2-4) was positive $(\mathrm{r}=0.193, \mathrm{P}<0.05)$ between final weight and $\mathrm{Ca} / \mathrm{P}$ ratio. Relationship (correlation) between weight gain and $\mathrm{Ca} / \mathrm{P}$ ratio (Table 5) showed a

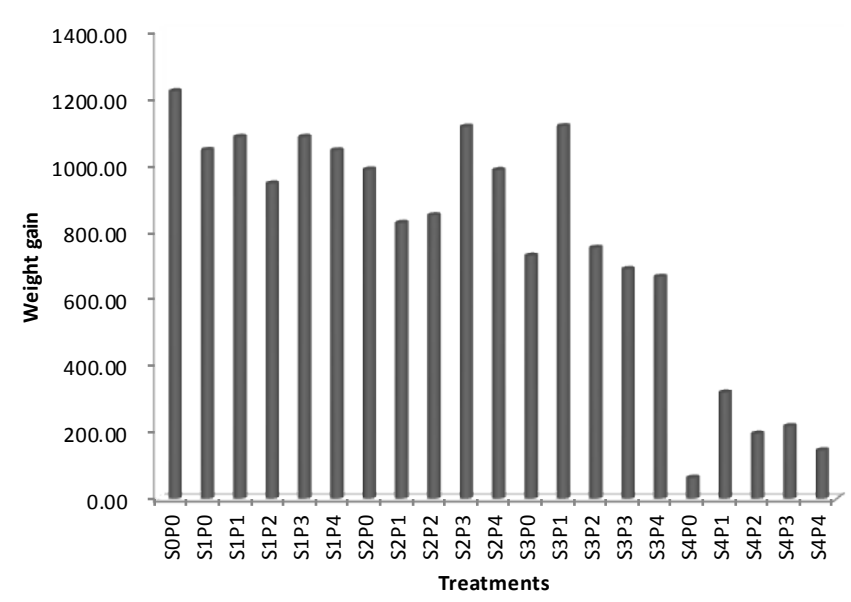

Figure 1: Weight gain of fish fed phytase-treated diet based on full-fat soyabean meal.

negative correlation $(\mathrm{r}=-0.431)$, while regression coefficient (Table 5 , Figure 5) was positive $\left(\mathrm{r}^{2}=0.186, \mathrm{P}<0.05\right)(\mathrm{P}<0.05)$ with $\mathrm{Ca} / \mathrm{P}$ ratio. Fish fed $250 \mathrm{FTU} / \mathrm{g}$ phytase had the highest weight gain $(\mathrm{P}>0.05)$, compared 
Citation: Akpoilih BU, Ajani EK, Omitoyin BO (2016) Optimum Dietary Ca/P Ratio and Phytase for Growth and Bone Mineralization in Juvenile Clarias gariepinus Fed Soya Bean-Based Diet. J Aquac Res Development 7: 403. doi:10.4172/2155-9546.1000403

Table 5: Relationship between calcium-phosphorus ratio, growth and nutrient utilization parameters.

\begin{tabular}{|c|c|c|c|c|c|}
\hline Parameters & a (Intercept) & b (Slope) & $\begin{array}{c}\mathbf{r}^{2} \text { (Regression } \\
\text { coefficient) }\end{array}$ & $\begin{array}{c}\text { r (Correlation } \\
\text { coefficient) }\end{array}$ & Sig. \\
\hline Final weight & 2229.287 & -754.226 & 0.193 & 0.439 & 0.004 \\
\hline Weight gained & 1899.961 & -740.001 & 0.186 & 0.431 & 0.004 \\
\hline Feed conversion ratio & -2.537 & 3.471 & 0.048 & 0.219 & 0.138 \\
\hline Specific growth rate & 1.928 & -0.330 & 0.019 & 0.05 & 0.191 \\
\hline Survival rate & 106.691 & -10.110 & 0.037 & $>0.05$ & 0.225 \\
\hline
\end{tabular}

The independent variable is the Ca-P ratio

The dependent variables are the Growth and Nutrient Utilization Parameters

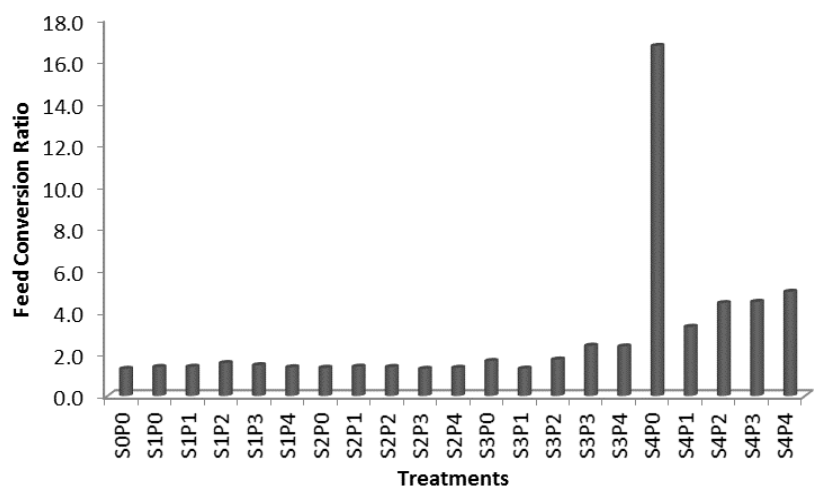

Figure 2: Feed conversion ratio of fish fed phytase-treated diet based on full-fat soyabean meal.

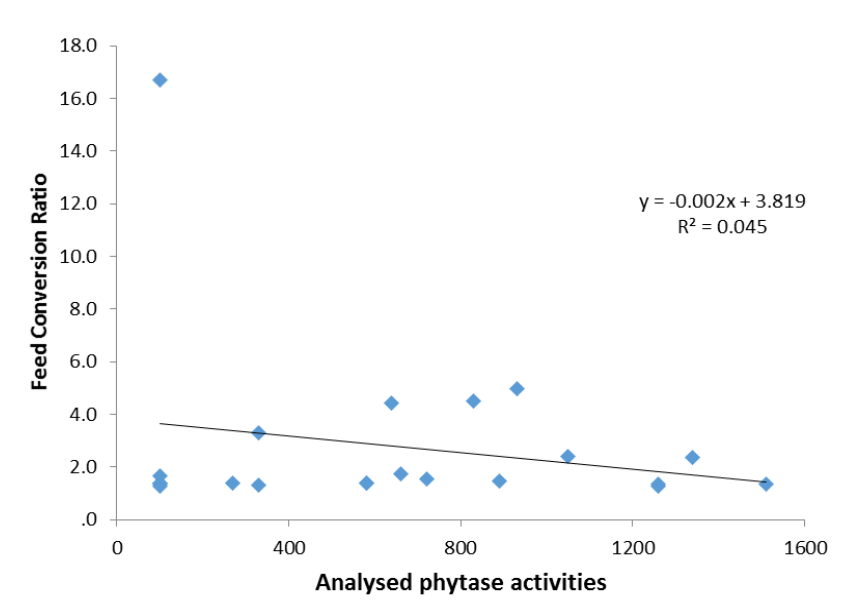

Figure 3: Relationship between analyzed phytase activity and feed conversion ratio.

to other phytase levels (Table 4). There was a statistically significant difference $(\mathrm{P}<0.05)$ in the feed conversion ratio of fish fed experimental diets (Table 3), which ranged between $1.29 \pm 0.01$ (S0P0) and $16.71 \pm$ 1.43 ( $\mathrm{S} 4 \mathrm{P} 0)$. There was no significant difference $(\mathrm{P}>0.05)$ between FCR values of fish fed S4P2 (4.43 \pm 0.06$)$, S4P3 (4.49 \pm 0.54$)$, and S4P4 (4.97 \pm 0.48 ). The lowest FCR value for fish fed $75 \%$ soya bean with phytase was recorded for S3P1 (1.41 \pm 0.01$)$, while the highest was S3P3 $(2.40$ $\pm 0.18)(\mathrm{P}<0.05)$. There is no significant difference between FCR of fish fed S3P1, S0P0, S1P0, S1P1, S1P1, S1P3, S1P4, S2P0, S2P1, S2P2, S2P3, $\mathrm{S} 2 \mathrm{P} 4(\mathrm{P}>0.05)$. Feed conversion by fish fed soya bean (full fat) diet

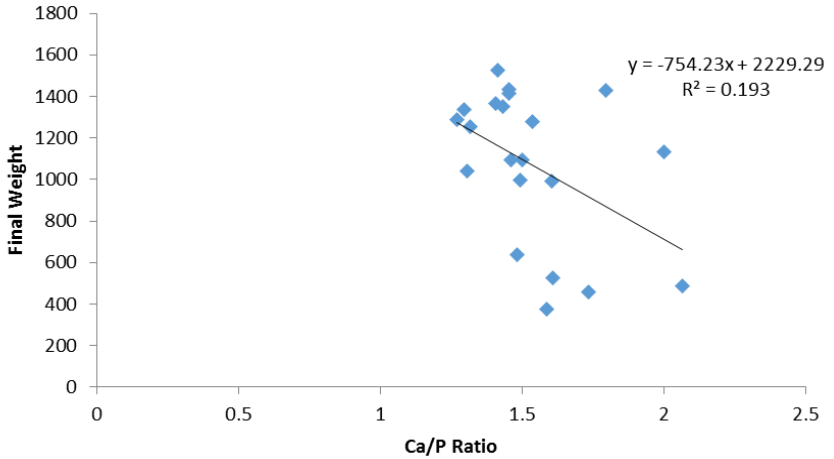

Figure 4: Relationship between $\mathrm{Ca} / \mathrm{P}$ ratio and final weight.

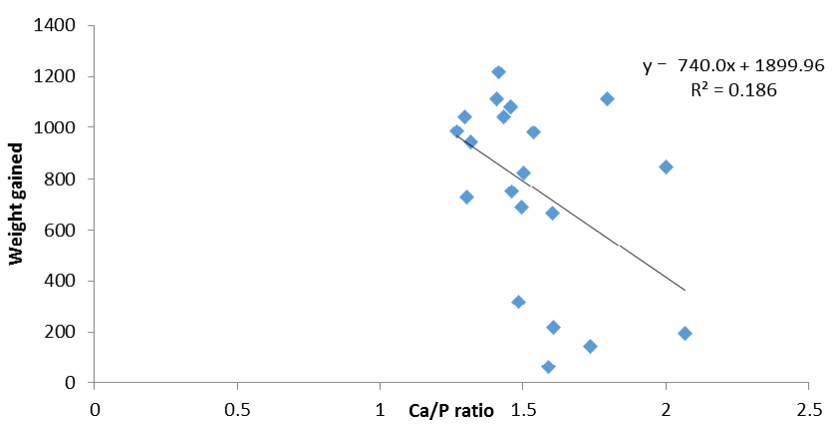

Figure 5: Relationship between $\mathrm{Ca} / \mathrm{P}$ ratio and weight gain.

with phytase is shown in Figure 2. In Table 4, phytase addition to diet at $250 \mathrm{FTU} / \mathrm{g}$ showed the lowest FCR value $(1.85 \pm 0.91)$, compared to $0 \mathrm{FTU} / \mathrm{g}(4.48 \pm 6.47), 500 \mathrm{FTU} / \mathrm{g}(2.28 \pm 1.34), 750 \mathrm{FTU} / \mathrm{g}(2.41$ $\pm 1.38)$, and $1000 \mathrm{FTU} / \mathrm{g}$ phytase $(2.51 \pm 1.60)(\mathrm{P}>0.05)$. Correlation $(\mathrm{r}=0.219, \mathrm{P}>0.05)$ and regression $\left(\mathrm{R}^{2}=0.048, \mathrm{P}>0.05\right)$ are positive between FCR and $\mathrm{Ca} / \mathrm{P}$ ratio. Specific growth rate was highest for fish fed S3P1 and the lowest in fish fed S4P0. Correlation and regression coefficients between SGR and $\mathrm{Ca} / \mathrm{P}$ ratio were $(\mathrm{r}=-0.138, \mathrm{P}>0.05)$ and $\left(r^{2}=0.019, P>0.05\right)$, respectively. Calcium/phosphorus ration was significantly affected by phytase supplementation in experimental diets $(\mathrm{P}<0.05)$. Values ranged from $1.27 \pm 0.02(\mathrm{~S} 2 \mathrm{P} 0)$ to $2.07 \pm 0.13(\mathrm{~S} 4 \mathrm{P} 2)$. Survival rate (Table 3 ) of fish showed a significant decrease in value with increasing levels of soya bean in basal diet $(\mathrm{P}<0.05)$. A significant decrease in survival rate was observed when phytase was supplemented in diet based on $25 \%$ and $75 \%$ soya bean with phytase $(\mathrm{P}<0.05)$. Fish fed S3P1 showed the lowest survival of fish $(73.08 \pm 10.88 \%)$ compared 
Table 6: Growth and nutrient utilization in juvenile Clarias gariepinus suboptimal phytase diet based on soyabean meal (full fat).

\begin{tabular}{|c|c|c|c|c|c|c|c|}
\hline Trt & Initial weight (g) & final weight (g) & weight gain (g) & SGR (\%) & FCR & Survival rate (g) & $\mathrm{Ca} / \mathrm{P}$ \\
\hline SOPO & $309.09 \pm 14.38$ & $1526.56 \pm 64.78^{g}$ & $1219.47 \pm 47.57^{f}$ & $1.90 \pm 0.01^{\mathrm{a}}$ & $1.29 \pm 0.01^{\text {ef }}$ & $92.31 \pm 0.00$ & 1.41 \\
\hline $\mathrm{S}_{3} \mathrm{D}$ & $383.14 \pm 2.92^{\mathrm{a}}$ & $606.81 \pm 99.29^{a}$ & $223.68 \pm 102.21^{a}$ & $0.55 \pm 0.21^{a}$ & $7.34 \pm 3.50^{a}$ & $93.0 \pm 2.0^{a}$ & 2.95 \\
\hline $\mathrm{S}_{3} \mathrm{P}_{50}$ & $372.71 \pm 38.47^{\mathrm{a}}$ & $581.68 \pm 40.50^{a}$ & $208.98 \pm 78.98^{a}$ & $0.54 \pm 0.21^{a}$ & $7.03 \pm 2.68^{a}$ & $96.5 \pm 1.5^{\mathrm{a}}$ & 1.61 \\
\hline $\mathrm{S}_{3} \mathrm{P}_{100}$ & $367.19 \pm 67.81^{\mathrm{a}}$ & $555.55 \pm 237.36^{a}$ & $188.36 \pm 169.55^{a}$ & $0.44 \pm 0.30^{a}$ & $10.70 \pm 7.97^{a}$ & $95.0 \pm 3.0^{\mathrm{a}}$ & 1.78 \\
\hline $\mathrm{S}_{3} \mathrm{P}_{150}$ & $383.16 \pm 49.92^{\mathrm{a}}$ & $518.64 \pm 155.01^{\mathrm{a}}$ & $135.73 \pm 104.74^{a}$ & $0.33 \pm 0.20^{a}$ & $13.45 \pm 9.22^{a}$ & $95.0 \pm 4.95^{\mathrm{a}}$ & 1.76 \\
\hline $\mathrm{S}_{3} \mathrm{P}_{200}$ & $356.94 \pm 30.67^{a}$ & $493.61 \pm 48.42^{a}$ & $137.68 \pm 19.17^{\mathrm{a}}$ & $0.39 \pm 0.01^{a}$ & $9.33 \pm 0.69^{a}$ & $98.0 \pm 0.0^{a}$ & 1.98 \\
\hline Sig & $P>0.05$ & $P>0.05$ & $P>0.05$ & $P>0.05$ & $P>0.05$ & $P>0.05$ & \\
\hline
\end{tabular}

Mean values with the same alphabet superscript in the same column are not significantly different $(P>0.05)(2$-tailed). $n=2$

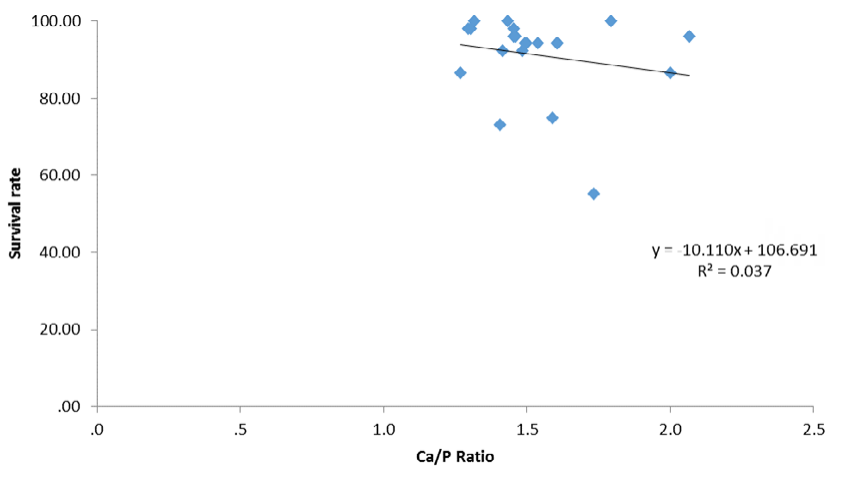

Figure 6: Relationship between $\mathrm{Ca} / \mathrm{P}$ ratio and survival rate.

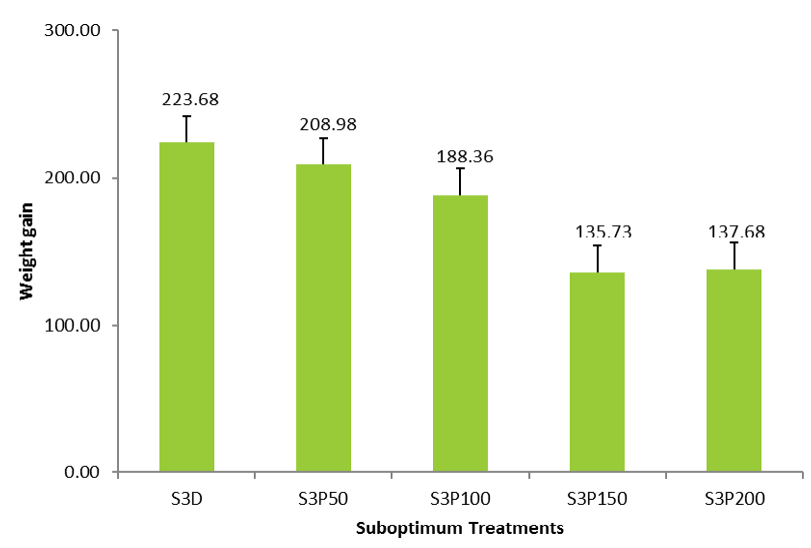

Figure 7: Weight gain of suboptimal phytase diet.

to S3P0 (98.08 $\pm 2.72 \%)$, S3P2 (96.15 $\pm 0.00 \%)$, S3P3 $(94.23 \pm 2.72 \%)$ and S3P4 (94.23 $\pm 2.72 \%)$. Fish fed $100 \%$ soya bean diet supplemented with 250, 500 and $750 \mathrm{FTU} / \mathrm{g}$ phytase had significantly higher values $(\mathrm{P}<0.05)$ of survival compared to control diet $(\mathrm{S} 4 \mathrm{P} 0)$. Regression analysis (Figure 6) showed a negative relationship between $\mathrm{Ca} / \mathrm{P}$ ratio and survival rate of fish $(\mathrm{P}>0.05)$. When fish were fed suboptimal phytase (less than $250 \mathrm{FTU} / \mathrm{g}$ ) diet between $50 \mathrm{FTU} / \mathrm{g}$ and $200 \mathrm{FTU}$ FTU/g in $75 \%$ soya bean meal diet, there were significant reduction in growth and nutrient utilization parameters (Table 6) compared to phytase-adequate diet with phytase at $250 \mathrm{FTU} / \mathrm{g}$ suggesting optimum is $250 \mathrm{FTU} / \mathrm{g}$. There was a significant increase in $\mathrm{Ca} / \mathrm{P}$ ratio in diets based on soya bean meal, which increased from $1.27 \pm 0.02$ in diet S2P0 to $1.59 \pm 0.04$ in S4P0. Addition of phytase resulted in a significant reduction in $\mathrm{Ca} / \mathrm{P}$ ratio of diets of fish fed $25 \%$ soya bean $(\mathrm{P}<0.05)$ from
$1.43 \pm 0.00(\mathrm{~S} 1 \mathrm{P} 0)$ to $1.29 \pm 0.02(\mathrm{~S} 1 \mathrm{P} 4)(\mathrm{P}<0.05)$. Diet of fish fed S3P1 had the lowest $\mathrm{Ca} / \mathrm{P}$ ratio $(1.41 \pm 0.02)$, compared to $\mathrm{Ca} / \mathrm{P}$ ratios of $\mathrm{S} 3 \mathrm{P} 2(1.46 \pm 0.00), \mathrm{S} 3 \mathrm{P} 3(1.49 \pm 0.02)$, and S3P4 $(1.60 \pm 0.02) . \mathrm{Ca} / \mathrm{P}$ is of S3P1 is not different $(\mathrm{P}>0.05)$ from S0P0, S1P0, S1P1, and S1P3) $(\mathrm{P}>0.05)$. Fish fed diets supplemented with $250 \mathrm{FTU} / \mathrm{g}(1.46 \pm 0.04)$ phytase had the lowest $\mathrm{Ca} / \mathrm{P}$ ratio compared to diets with 500 (1.71 \pm $0.35), 750(1.59 \pm 0.14)$, and $1000 \mathrm{FTU} / \mathrm{g}(1.54 \pm 0.17)(\mathrm{P}<0.05)($ Table 4 and Figure 7).

\section{Bone mineralization}

There was significant reduction $(\mathrm{P}<0.05)$ in bone phosphorus (Table 7) in diet without phytase from $4.50 \pm 0.01 \%$ in S1P0 to $2.33 \pm$ $0.01 \%$ in S4P0. Values of phosphorus, however, increased significantly in $25 \%$ soya bean diet for fish fed S1P1-S1P3 and in 50\% soya bean for S2P1 and S2P2 fish. In fish fed 75\% soya bean diet with phytase addition, there was no difference between S3P0 and S3P4 fish $(\mathrm{P}>0.05)$. However, there were significant increase in bone phosphorus when fish were fed $100 \%$ soya bean diet with phytase from $2.33 \pm 0.01 \%$ in $\mathrm{S} 4 \mathrm{P} 0$ to $2.89 \pm 0.01 \%$ in $\mathrm{S} 4 \mathrm{P} 4(\mathrm{P}<0.05)$. S0P0 fish had the highest phosphorus (5.74 $\pm 0.01 \%)$. Table 8 shows that phosphorus was highest in $250 \mathrm{FTU} / \mathrm{g}$ compared to other phytase levels. Fish fed experimental diet showed significant differences in bone calcium $(\mathrm{P}<0.05)$. Levels of calcium decreased significantly in soya bean diet with phytase from $2.33 \pm 0.01 \%$ in fish fed S1P0 to $1.41 \pm 0.01 \%$ in S4P0. Addition of phytase significantly increased calcium levels in fish fed $25 \%$ and $100 \%$ soya bean $(\mathrm{P}<0.05)$. Values of calcium also increased in fish fed $75 \%$ soya bean, which was, however, not significant $(\mathrm{P}>0.05)$. The lowest recorded bone calcium for experimental fish was in fish fed S4P0 (1.41 $\pm 0.01 \%)$, while fish fed S0P0 had the highest level of bone calcium $(2.89 \pm 0.01 \%)$. Bone zinc improved significantly in all diets compared to diets without phytase. Like phosphorus and calcium, values were higher for $250 \mathrm{FTU} / \mathrm{g}$ in fish fed $75 \%$ soya bean, compared to 0 , 500, 750 and $1000 \mathrm{FTU} / \mathrm{g}$ phytase (Table 8, suggesting $250 \mathrm{FTU} / \mathrm{g}$ is optimum for both growth and bone mineralization in juvenile Clarias gariepinus. Phosphorus retention was highest in fish fed $75 \%$ soya bean with $250 \mathrm{FTU} / \mathrm{g}$ (Table 7) and was highest $(0.73 \pm 0.23)$ compared to 0 FTU $(0.53 \pm 0.54), 500$ FTU $(0.58 \pm 0.25), 750$ FTU $(0.57 \pm 0.20)$, and 1000 FTU $(0.58 \pm 0.15)$ (Table 8$)$.

\section{Discussion}

\section{Mineral composition of the diet}

Phytase addition to fish diet has been reported to increase dietary mineral retention in fish [8,34-36]. In this experiment, significant effect of phytase on mineral availability was observed. Although phosphorus in $25 \%$ soya bean and $50 \%$ soya bean (full fat) were sufficient for fish in meeting the requirement for the mineral $(0.67-0.82 \%)$ as determined by Nwanna et al. [37], the significant increase in dietary phosphorus by phytase in the former could be related to phytate hydrolysis and 
Table 7: Bone mineralization and phosphorus retention efficiency of fish fed phytase-treated experimental diet based on soyabean meal (full fat).

\begin{tabular}{|c|c|c|c|c|c|c|}
\hline Treatment & Phosphorus (\%) & Calcium (\%) & Magnesium (\%) & Iron (ppm) & Zinc (ppm) & $\begin{array}{c}\text { Phosphorus retention } \\
\text { efficiency (\%) }\end{array}$ \\
\hline Initial fish & $2.21 \pm 0.01$ & $2.59 \pm 0.01$ & $0.17 \pm 0.01$ & $7.29 \pm 0.01$ & $28.77 \pm 0.01$ & \\
\hline SOPO & $5.74 \pm 0.01^{q}$ & $2.89 \pm 0.01^{k}$ & $0.25 \pm 0.01^{\mathrm{h}}$ & $5.89 \pm 0.01^{r}$ & $27.41 \pm 0.01^{d}$ & $0.62 \pm 0.01^{i}$ \\
\hline S1P0 & $4.50 \pm 0.01^{j}$ & $2.33 \pm 0.01^{\text {gh }}$ & $0.22 \pm 0.01^{g}$ & $5.54 \pm 0.02^{p}$ & $24.71 \pm 0.01^{b}$ & $0.62 \pm 0.01^{\mathrm{hi}}$ \\
\hline S1P1 & $5.68 \pm 0.01^{p}$ & $2.85 \pm 0.01^{\mathrm{jk}}$ & $0.18 \pm 0.01^{\text {ef }}$ & $4.49 \pm 0.01^{\prime}$ & $28.81 \pm 0.01^{f}$ & $0.53 \pm 0.01^{f}$ \\
\hline S1P2 & $5.30 \pm 0.01^{n}$ & $2.50 \pm 0.01^{i}$ & $0.20 \pm 0.01^{\mathrm{fg}}$ & $6.45 \pm 0.01^{\mathrm{t}}$ & $29.34 \pm 0.01^{i}$ & $0.54 \pm 0.01^{f}$ \\
\hline S1P3 & $5.13 \pm 0.01^{m}$ & $2.72 \pm 0.01^{j}$ & $0.26 \pm 0.01^{\mathrm{h}}$ & $5.10 \pm 0.01^{n}$ & $35.03 \pm 0.01^{p}$ & $0.45 \pm 0.01^{\mathrm{e}}$ \\
\hline S1P4 & $4.14 \pm 0.01^{i}$ & $2.53 \pm 0.01^{i}$ & $0.25 \pm 0.01^{\mathrm{h}}$ & $5.23 \pm 0.01^{\circ}$ & $28.21 \pm 0.01^{e}$ & $0.70 \pm 0.01^{k}$ \\
\hline S2P0 & $4.89 \pm 0.01^{1}$ & $2.19 \pm 0.01^{\mathrm{fg}}$ & $0.11 \pm 0.01^{a}$ & $6.32 \pm 0.01^{\mathrm{s}}$ & $32.19 \pm 0.01^{m}$ & $0.78 \pm 0.01^{m}$ \\
\hline $\mathrm{S} 2 \mathrm{P} 1$ & $5.28 \pm 0.01^{n}$ & $2.49 \pm 0.01^{\mathrm{hi}}$ & $0.20 \pm 0.01^{\mathrm{fg}}$ & $4.15 \pm 0.01^{j}$ & $32.16 \pm 0.01^{\mathrm{m}}$ & $0.72 \pm 0.01^{\mathrm{kl}}$ \\
\hline S2P2 & $5.60 \pm 0.01^{\circ}$ & $2.49 \pm 0.35^{\mathrm{hi}}$ & $0.25 \pm 0.01^{\mathrm{h}}$ & $7.98 \pm 0.01^{u}$ & $29.28 \pm 0.04^{h}$ & $0.92 \pm 0.01^{\circ}$ \\
\hline S2P3 & $4.69 \pm 0.01^{k}$ & $2.05 \pm 0.01^{\text {ef }}$ & $0.15 \pm 0.01^{\text {cd }}$ & $3.09 \pm 0.01^{g}$ & $22.79 \pm 0.01^{a}$ & $0.82 \pm 0.01^{n}$ \\
\hline $\mathrm{S} 2 \mathrm{P} 4$ & $3.78 \pm 0.01^{\mathrm{h}}$ & $1.65 \pm 0.01^{b c}$ & $0.17 \pm 0.01^{\mathrm{de}}$ & $2.08 \pm 0.01^{\mathrm{e}}$ & $34.28 \pm 0.04^{\circ}$ & $0.74 \pm 0.01^{1}$ \\
\hline S3P0 & $3.42 \pm 0.01^{\mathrm{g}}$ & $2.23 \pm 0.01^{\mathrm{g}}$ & $0.19 \pm 0.01^{\text {ef }}$ & $4.23 \pm 0.01^{k}$ & $31.19 \pm 0.01^{j}$ & $1.07 \pm 0.01^{p}$ \\
\hline S3P1 & $3.26 \pm 0.01^{\mathrm{e}}$ & $1.97 \pm 0.01^{\mathrm{e}}$ & $0.15 \pm 0.01^{c d}$ & $2.31 \pm 0.01^{f}$ & $32.62 \pm 0.01^{n}$ & $1.08 \pm 0.03^{p}$ \\
\hline S3P2 & $3.13 \pm 0.01^{d}$ & $1.88 \pm 0.01^{\mathrm{de}}$ & $0.14 \pm 0.01^{\mathrm{bcd}}$ & $3.35 \pm 0.01^{\mathrm{h}}$ & $27.44 \pm 0.01^{d}$ & $0.60 \pm 0.01^{\text {gh }}$ \\
\hline S3P3 & $3.42 \pm 0.01^{g}$ & $1.98 \pm 0.01^{\mathrm{e}}$ & $0.17 \pm 0.01^{d e}$ & $4.61 \pm 0.01^{m}$ & $36.59 \pm 0.01^{\mathrm{s}}$ & $0.35 \pm 0.01^{c}$ \\
\hline S3P4 & $3.37 \pm 0.01^{f}$ & $2.25 \pm 0.01^{\mathrm{g}}$ & $0.31 \pm 0.01^{i}$ & $5.74 \pm 0.01^{q}$ & $31.73 \pm 0.01^{\prime}$ & $0.46 \pm 0.01^{\mathrm{e}}$ \\
\hline S4P0 & $2.33 \pm 0.01^{a}$ & $1.41 \pm 0.01^{a}$ & $0.12 \pm 0.01^{a b}$ & $0.67 \pm 0.01^{a}$ & $31.38 \pm 0.01^{k}$ & $-0.44 \pm 0.01^{a}$ \\
\hline S4P1 & $3.42 \pm 0.01^{g}$ & $1.74 \pm 0.01^{\mathrm{cd}}$ & $0.14 \pm 0.01^{\mathrm{abc}}$ & $3.51 \pm 0.01^{i}$ & $37.29 \pm 0.01^{q}$ & $0.59 \pm 0.01^{g}$ \\
\hline S4P2 & $2.66 \pm 0.01^{b}$ & $1.64 \pm 0.01^{b c}$ & $0.14 \pm 0.01^{\mathrm{bcd}}$ & $1.05 \pm 0.01^{b}$ & $38.09 \pm 0.01^{r}$ & $0.26 \pm 0.01^{b}$ \\
\hline S4P3 & $2.64 \pm 0.01^{b}$ & $1.56 \pm 0.01^{b}$ & $0.25 \pm 0.01^{\mathrm{h}}$ & $1.61 \pm 0.01^{d}$ & $29.04 \pm 0.01^{g}$ & $0.67 \pm 0.01^{j}$ \\
\hline S4P4 & $2.89 \pm 0.01^{c}$ & $1.50 \pm 0.01^{b}$ & $0.11 \pm 0.01^{a}$ & $1.22 \pm 0.01^{c}$ & $27.37 \pm 0.01^{c}$ & $0.42 \pm 0.01^{d}$ \\
\hline Total & $4.06 \pm 1.10$ & $2.13 \pm 0.45$ & $0.19 \pm 0.06$ & $4.03 \pm 1.99$ & $35.57 \pm 23.16$ & $0.59 \pm 0.31$ \\
\hline Sig & $P<0.05$ & $P<0.05$ & $P<0.05$ & $P<0.05$ & $P<0.05$ & $P<0.05$ \\
\hline
\end{tabular}

Mean values with the same alphabet superscript in the same column are not significantly different at the 0.05 level (2-tailed)

Table 8: Effect of phytase on bone minerals and phosphorus retention efficiency in fish fed soybean diet.

\begin{tabular}{|c|c|c|c|c|c|}
\hline Phytase & Phosphorus (\%) & Calcium (\%) & Magnesium (\%) & Iron (ppm) & Zhosphorus (ppm) \\
retention efficiency
\end{tabular}

breakdown, which made phosphorus available (Table 2) in these diet $[5,6,8]$ in excess of requirement [14]. While dietary phosphorus declined in diet with $50 \%$ full fat soya bean with increasing phytase addition from $250 \mathrm{FTU} / \mathrm{g}$ to $1000 \mathrm{FTU} / \mathrm{g}$ (Table 2), values of available phosphorus increased with $1000 \mathrm{FTU} / \mathrm{g}$ phytase. Increase in available phosphorus in both diets could be due to high phytate (Table 1) in $25 \%(0.52 \%)$ and $50 \%$ soya bean $(0.57 \%)$ without phytase, compared to phytate levels in $75 \%(0.43 \%)$ and $100 \%(0.40 \%)$ full fat soya bean diet. The magnitude of response to phytase was greater in ingredients containing high level of phytate for basal diet with $25 \%$ and $50 \%$ soya bean with phytase supplementation compared to $75 \%$ and $100 \%$ full fat soya bean diet $[6,38]$. Available phosphorus levels (Table 2) in $75 \%$ full fat soya bean with $250 \mathrm{FTU} / \mathrm{g}$ (S3P1) was highest compared to values in other diets (S3P0, S3P2, S3P3, S3P4), which indicated that phytase at that level significantly increased phytate hydrolysis [6] and thus increasing the level of available phosphorus from $0.33 \pm 0.01 \%$ in control S3P0 to $0.42 \pm 0.02 \%$, which was above the requirement $(0.33 \%-0.40 \%)$ for catfish [13,39]. Values of available phosphorus in $100 \%$ soya bean diet (full fat) with phytase showed a significant increase and could be due increased phytate utilization even at lowest level of dietary phosphorus compared to other dietary soya bean levels $[6,40]$. Although, levels of calcium were above the requirement of $0.45 \%$ for catfish [24], calcium increased with phytase in all diet except in $25 \%$ soya bean. Increase in calcium could be due to phytase effect, which released calcium from calcium-phytate complex and the presence of high phytate in these diets $[6,38]$, thus making it available in the diet [23]. Phytase has little effect in diet when phytate content is less than $2.0 \mathrm{~g} / \mathrm{kg}$ diet [6]. Increase in dietary zinc is associated with phytaseinduced breakdown of phytate-zinc complex [6], thus improving utilization of phytate-bound minerals, especially phosphorus and zinc.

\section{Growth and nutrient utilization}

Phytase has been extensively used in animal nutrition to enhance growth and feed and nutrient utilization [19,37,38,41]. Phytase enhanced apparent digestibility of minerals and their deposition in the fish [20]. It increased mineral utilization through the breakdown of phytate-mineral complex [23], which resulted in increased growth and feed efficiency of the fish $[7,37]$ through improvement in phosphorus bioavailability [23]. Improved growth performance and nutrient 
utilization were observed in this research investigating the possibility of substituting entirely or almost entirely level of fish meal in fish diet [7]. Phytase addition at 250, 500, 750, and $1000 \mathrm{FTU} / \mathrm{g}$ to $25 \%$ full fat soya bean meal recorded positive weight gain (Table 3 ) compared to diet without phytase (25\% soya bean, no phytase). When fish meal was substituted with $50 \%$ soya bean (full fat), positive effect was observed with $750 \mathrm{FTU} / \mathrm{g}$ compared to other diets (S2P1, S2P2, S2P4), and was comparable to weight gain of fish fed control diet (SOP0). At higher level of fish substitution by soya bean (full fat), phytase inclusion at 250 FTU/g was better than at $0,500,750$, and $1000 \mathrm{FTU} / \mathrm{g}$.

Weight gain at $250 \mathrm{FTU} / \mathrm{g}$ in $75 \%$ soya bean (full fat) was better than at lower fish meal substitution $(25 \%$ and $50 \%)$ by soya bean (full fat) with phytase addition at any level, and performed as fish fed control (100\% fish meal). This could be explained by fact that phytase is effective in hydrolyzing phytate at low dietary phosphorus and low available phosphorus level $[6,7,23]$. The total and available phosphorus in $75 \%$ soya bean diet (full fat) without phytase are much lower $(0.75 \pm$ $0.01 \%$ and $0.33 \pm 0.01 \%)$ than at $25 \%(1.15 \pm 0.01 \%$ and $0.63 \pm 0.01 \%)$ and 50\% (1.01 $\pm 0.01 \%$ and $0.44 \pm 0.01 \%)$. Denbow et al. [42] tried seven levels of supplemental phytase at $0,200,400,600,800,1000$, and $1200 \mathrm{FTU} / \mathrm{g}$ in the semi purified diet of broilers containing 0.20 , 0.27 or $0.34 \%$ available phosphorus. They observed improved body weight gain and feed intake $(\mathrm{P}<0.01)$ at all available phosphorus levels, but the magnitude of response was maximum at low available phosphorus level. Lim et al. [40] supplemented microbial phytase at $500 \mathrm{FTU} / \mathrm{g}$ in a corn-soya bean meal based broiler diet $(0.25 \%, 0.35 \%$ and $0.45 \%$ available phosphorus) and observed significant $(\mathrm{P}<0.01)$ improvements in body weight gain. They reported enhanced benefits of phytase in low available phosphorus diet, but no significant $(\mathrm{P}<0.01)$ effect in diets containing higher level of phytate phosphorus. Johnston and Southern (2000) used eight levels of phytase supplementation at 0 , $200,400,600,800,1000$, and $1200 \mathrm{FTU} / \mathrm{g}$ ) in low phytate phosphorus broiler diet and reported improvements in body weight gain, and that increased levels of phytase supplementation had little effect on growth performance $(\mathrm{P}<0.01)$. Phytase supplementation at $500 \mathrm{FTU} / \mathrm{g}$ to low available phosphorus $(0.30 \%)$ diets significantly $(\mathrm{P}<0.05)$ improved the growth performance of broilers. This was also observed in improved growth performance in fish fed with phytase $(250 \mathrm{FTU} / \mathrm{g})$ soya bean diet $(75 \%)$ with low basal available phosphorus $(0.33 \pm$ $0.01 \mathrm{~g}$ ) compared with fish fed $25 \%$ and $50 \%$ soya bean with available phosphorus of $0.63 \pm 0.01 \%$ and $0.44 \pm 0.01 \%$, respectively (Table 2). Additionally, fish fed $75 \%$ soya bean with $250 \mathrm{FTU} / \mathrm{g}$ showed the best growth performance and nutrient utilization, which was reported to be best by Castro et al. who reported $75 \%$ soya bean with phytase (4,000 FTU/g, Ronozyme) as best for Rainbow trout. In the study of $\mathrm{Li}$ and Robinson, fish fed the diets containing $250 \mathrm{FTU} / \mathrm{g}$ phytase or above consumed more feed, gained more weight, and had a lower feed conversion ratio in comparison to fish fed the basal diet containing no microbial phytase. In Li et al., phytase supplementation at $250 \mathrm{FTU} / \mathrm{g}$ in catfish diet increased feed intake, body weight gain, feed efficiency, and bone ash and phosphorus concentration. In the present research, $250 \mathrm{FTU} / \mathrm{g}$ at $75 \%$ soya bean and at all levels of soya bean (irrespective of soya bean levels, Table 4), had the highest specific growth rate, weight gain, and lowest feed conversion compared with others levels of soya bean diet (full fat). Feed conversion declined with increasing phytase as seen from the regression graph between FCR and analyzed phytase activity (Figure 3), which means weight gain is optimum or highest with low phytase. In Li and Robinson, channel catfish fed the diets containing $250 \mathrm{FTU} / \mathrm{g}$ or above consumed more feed, gained more weight, and had a lower feed conversion ratio in comparison to fish fed the basal diet containing no microbial phytase. Robinson et al. reported that phytase at $250 \mathrm{FTU} / \mathrm{g}$ could effectively replace dicalcium phosphate supplement in the diet of channel catfish without affecting growth, feed efficiency or bone $\mathrm{P}$ deposition. This was also observed in this experiment in which diets with $250 \mathrm{FTU} / \mathrm{g}$ at $75 \%$ soya bean (S3P1) had better growth performance (Table 2) compared to positive control, $\mathrm{S}_{3} \mathrm{D}$ (Table 6), suggesting phytase at this level can effectively replace inorganic phosphorus in Clarias gariepinus diet. Fish fed suboptimal phytase diet (Table 6) showed poor growth performance compared to diet with optimal level of phytase at $250 \mathrm{FTU} / \mathrm{g}$ (Table 3), which could be due to low phosphorus availability and high $\mathrm{Ca} / \mathrm{P}$ ratios in the diet (Table 6) compared to S3P1 and S0P0 (Table 3).

Fish fed $100 \%$ soya bean diet (full fat) showed the poorest growth response compared to other soya bean levels, although there were significant improvement in weight gain compared to control (S4P0). The low growth in $100 \%$ soya bean fish could be related to unbalanced amino acid profile of soya bean compared to fish meal [43] limiting its total substitution of fish meal as well as the high level of fat, which was beyond the maximum level that can be used in fish feed [24]. Fish fed $100 \%$ soya bean also showed the lowest feed intake, specific growth rate, protein efficiency, and the highest feed conversion. 100\% soya bean diet (full fat) also showed a significantly higher calcium/phosphorus ratio, which depresses phytate hydrolysis and reduce growth [23]. The Ca:P of $75 \%$ soya bean meal supplemented with $250 \mathrm{FTU} / \mathrm{g}(1.41: 1)$ is comparable to that of $100 \%$ fish meal diet (1.41:1), suggesting optimum level in soya bean diet (full fat) for Clarias gariepinus fish diet. In Table 4, phytase at $250 \mathrm{FTU} / \mathrm{g}$ gave the highest growth response with optimum $\mathrm{Ca} / \mathrm{P}$ of 1.46 compared to phytase at $0 \mathrm{FTU} / \mathrm{g}(\mathrm{Ca} / \mathrm{P}=1.40)$, $500 \mathrm{FTU} / \mathrm{g}(\mathrm{Ca} / \mathrm{P}=1.71), 750 \mathrm{FTU} / \mathrm{g}(\mathrm{Ca} / \mathrm{P}=1.59)$ and $1000 \mathrm{FTU} / \mathrm{g}(\mathrm{Ca} /$ $\mathrm{P}=1.54$ ) (Table 6). In Kim et al. [44], growth performance and nutrient utilization in weaning pig fed phytase-supplemented-canola-soya bean diet with high phytate and $\mathrm{Ca} / \mathrm{P}$ of 1.46 was significantly higher than diets without phytase. Cao et al. [7] recommended a range of Ca:P between 1.1:1 and 1.4:1 for optimum phytase activity in fish feed. The high Ca:P in S2P1 (1.54), S2P2 (2.00) and S2P4 (1.54) as well as the high level in $100 \%$ soya bean with phytase, in the diet could be responsible for the poor weight gain of fish fed these diets compared to S0P0, S2P0, S2P3, S3P1 and S3P4. Ca:P ratios are crucial to phytase efficacy as reviewed by Lei and Stahl [22], since high level chelate with phytate and compete with active site of phytase, which reduces phytase efficacy and phosphorus availability [1]. Qian et al. reported that reducing Ca:P ratios from 2.0 to 1.2:1 in phytase supplemented diets (700 and 1050 FTU $/ \mathrm{kg}$ ) of pig, increased phytase efficacy by the order of $16 \%$. Liu et al. observed that reducing Ca:P ratios from 1.5 to 1.0:1 in phytase (500 FTU/kg) supplemented low phosphorus diets increased P absorption in the small intestine of pigs. Similarly, Qian et al. found that increasing Calcium (5.61-10.20 g/kg) and Ca:P ratios (1.1-2.0:1) depressed weight gain of broilers

\section{Bone mineralization}

The effect of phytase supplementation in diet fish has been shown improve bone mineralization in several studies $[1,7,9]$ Channel catfish fed phytase-supplemented diets had higher concentrations of ash, calcium, phosphorus and manganese in their bones than the fish fed on a control diet. Bone mineralization in Common carp was improved by phytase addition in the diet $[37,41]$.

In this study, phytase supplementation improved bone parameters, depending on the level of soya bean in the diet. Bone phosphorus, calcium, iron, and zinc (Table 7) were improved in fish fed 25 and $50 \%$ soya bean (full fat) with phytase supplementation at $250-750$ 
FTU/g and 250-500 FTU/g, respectively, compared to basal controls in these diets $[5,45]$. Increased mobilization of phosphorus store in soft tissue such as the flesh from fish fed these diets improved bone mineralization in these fish without depleting their store in the flesh, suggesting adequate phosphorus intake, contrary to who reported that insufficient phosphorus intake leads to the mobilization of phosphorus from the bone and transfer to soft tissues and metabolic processes [46]. According to Baruah et al. [5], dietary supplementation of microbial phytase at $500 \mathrm{FTU} / \mathrm{g}$ in Labeo rohita (Hamilton) juveniles' diet significantly increased bone sodium, calcium, potassium, manganese and iron content by 15, 12.1, 17.4, 20.4 and $40.7 \%$, respectively. Robinson et al. [14] reported that phytase of $250 \mathrm{FTU} / \mathrm{g}$ could effectively replace dicalcium phosphate supplement in the diet of channel catfish without affecting growth, feed efficiency or bone phosphorus deposition. In this study, fish fed $75 \%$ soya bean (full fat) with 250 FTU showed significant reduction in bone phosphorus and calcium, which could be due to inadequate supply $[36,46]$ fat in the diet. Reduction in bone phosphorus and calcium in $75 \%$ soya bean with $250 \mathrm{FTU} / \mathrm{g}$ could be due to inhibition of their absorption by fat in the diet for whole body retention and bone mineralization. However, in other diets (25\%,50\% and $100 \%$ soya bean), phytase at $250 \mathrm{FTU} / \mathrm{g}$ improved significantly bone phosphorus compared to controls without phytase. Reqz et al. reported that soyabean oil affected bone characteristics (bone length and volume) in animals. There was, however, significant increase in iron and zinc, indicating phytase effectively released zinc from zinc-phytate complex [23] for retention in whole body and bone mineralization. Improvement of zinc in diet and bone by supplementing phytase at $250 \mathrm{FTU} / \mathrm{g}$ could be the reason why it produced the greatest growth response in fish fed $75 \%$ soya bean meal diet compared to other phytase supplemented soya bean diet, and could effectively replace fish meal at that level of soya bean in the diet of juvenile Clarias gariepinus. Zinc exhibits a greater effect on growth than growth hormone and the nutrition of zinc for monogastric animals is similar physiologically to that of man. Most zinc deficiency is attributable to the abundance of dietary phytate, a natural compound found in all plant seeds and some roots and tubers. Fish fed $100 \%$ soya bean showed the greatest positive and consistent response to increasing phytase with the highest phosphorus at $250 \mathrm{FTU} / \mathrm{g}$ as observed in $25 \%$ and $50 \%$ soya bean, which could explained by low phosphorus level that enhances phytase response [40], and improving bone parameters. Irrespective of soyabean levels, fish fed diets with $250 \mathrm{FTU} / \mathrm{g}$ phytase showed the highest bone phosphorus and calcium compared to 500, 750 , and $1000 \mathrm{FTU} / \mathrm{g}$ (Table 8).

In conclusion, the research has demonstrated that phytase at $250 \mathrm{FTU} / \mathrm{g}$ was optimum for juvenile Clarias gariepinus as observed by improvement in growth (Figure 1), bone mineralization (Table 8 ) and phosphorus retention efficiency (Table 7) compared to other levels, and can replace dicalcium phosphate [14] however, the effect of $\mathrm{Ca} / \mathrm{P}$, from the research, has also shown the negative effect its negative effects at high levels as seen from the relationships between weight gain and total weight. Hence, it is recommended that, since the optimum phytase diet, which produced the best growth at $75 \%$ soya bean diet at $250 \mathrm{FTU} / \mathrm{g}(\mathrm{Ca} / \mathrm{P}$ for S3P1=1.41), which also gave the highest growth response $(\mathrm{Ca} / \mathrm{P}=1.46)$ compared to phytase at $0 \mathrm{FTU} / \mathrm{g}(\mathrm{Ca} / \mathrm{P}=1.40)$, $500 \mathrm{FTU} / \mathrm{g}(\mathrm{Ca} / \mathrm{P}=1.71), 750 \mathrm{FTU} / \mathrm{g}(\mathrm{Ca} / \mathrm{P}=1.59)$ and $1000 \mathrm{FTU} / \mathrm{g}(\mathrm{Ca} /$ $\mathrm{P}=1.54), \mathrm{Ca} / \mathrm{P}$ in the diet of juvenile Clarias gariepinus should range between 1.41-1.46 for optimum phytase activity. Available phosphorus should be as low as possible $(<0.33 \%)$.

\section{Acknowledgement}

Special thanks goes to Dr. Robert Ruehle for facilitating the supply of the enzyme from BASF company, Ludwigshafen, Germany; and his team of $R$ Tunnicliffe and Mrs. M. Ballandies (BASF, Lampertheim, Germany) for their technical, material and analytical support throughout the research.

\section{References}

1. Kumar V, Sinha AK, Makkar HPS, De Boeck G, Becker K (2011) Phytate and phytase in fish nutrition. J Anim Physiol Anim Nutr 96: 335-64.

2. http://faostat.fao.org/site/291/default.aspx

3. Cheeke PR (1971) Nutritional and physiological implications of saponins: A review. Can J Anim Sci 51: 621-632.

4. Jackson AJ, Copper BS, Matty AJ (1982) Evaluation of some plant protein In complete diet for the tilapia, Sarotheredon mossarnbicus. Aquaculture 27 97-109.

5. Baruah K, Pal AK, Sahu NP, Debnath D (2007) Microbial phytase supplementation in Rohu (Labeo rohita) diets enhances growth performance and nutrient digestibility. Journal of World Aquaculture Society 38: 129-137.

6. Selle PH, Ravindran V (2007) Microbial phytase in poultry nutrition. Anim Feed Sci Technol 135: 1-41.

7. Cao L, Wang W, Yang C, Yang Y, Diana J, et al. (2007) Application of microbial phytase in fish feed. Enz Microb Technol 14: 342-362.

8. Cain KD, Garling DL (1995) Pretreatment of soybean meal with phytase for Salmonid diets to reduce phosphorus concentration in hatchery effluents. Prog Fish-Cult 57: 114-119.

9. Nwanna LC (2007) Effect of Dietary phytase on Growth, Enzyme Activities and Phosphorus Load of Nile Tilapia (Oreochromis niloticus). Journal of Engineering and Applied Sciences 2: 972-976.

10. Hashim R (2006) Alternative ingredients for aquafeeds: the feasibility and economic equation. Paper presented as Guest Speaker at the National Fisheries Symposium (NaFIS), Kuching, Sarawak, Malaysia.

11. Leytem AB, Maguire RO (2007) Environmental implications of inosito phosphates in animal manures. In: Inositol Phosphates Linking Agriculture and the Environment (1st EDN) BL Turner, AE Richardson, EJ Mullaney, Cambridge, USA.

12. Lei XG, Porres JM (2003) Phytase enzymology, applications, and biotechnology Biotechnol Lett 25: 1787-94.

13. Robinson EH, Li MH (2006) Catfish nutrition: Nutrient requirements. Publication 2412 Extension Service of Mississippi State University.

14. Robinson EH, Li MH, Manning BB (2001) A Practical Guide to Nutrition, Feeds, and Feeding of Catfish (Second Revision). Agricultural communications bulletin 1113, Division of Agriculture, Forestry, and Veterinary Medicine, Mississipp State University. USA.

15. Furuya WM, Goncalves GS, Rossetto V, Furuya B, Hayashi C (2001) Phytase as feeding for Nile tilapia (Oreochromis niloticus): performance and digestibility. Revista Brasileira de Zootecnia 30: 924-929.

16. Yoo GY, Wang XJ, Choi SM, Han KM (2005) Dietary microbial phytase increase the phosphorus digestibility in juvenile Korean rockfish Sebastes schlegeli fed diets containing soybean meal. Aquaculture 243: 315-623.

17. Debnath D, Sahu NP, Pal AK, Jain KK, Yengkokpam S, Mukherjee SC (2005a) Mineral status of Pangasius pangasius (Hamilton) fingerlings in relation to supplemental phytase: absorption, whole body and bone mineral content Aquaculture Research. 36: 326-335.

18. Cheng ZJ, Hardy RW, Verlhac V, Gabaudan J (2004) Effects of microbial phytase supplementation and dosage on apparent digestibility coefficients of nutrients and dry matter in soybean product-based diets for rainbow trout. Journal of the World Aquaculture Society 35: 1-15.

19. VanWeerd JH, Khalaf KH, Aartsen EJ, Tijssen PA (1999) Balance trials with African catfish Clarias gariepinus fed phytase-treated soybean meal-based diets. Aquacult Nutr 5: 135-42.

20. Nwanna LC, Fagbenro OA, Adeyo AO (2005) Effects of different treatment of soyabean meal and phytase on the growth and mineral deposition in African catfish (Clarias gariepinus). Journal of Animal and Veterinary Advances 4: 980-987.

21. Angel R, Tamim NM, Applegate TJ, Dhandu AS, Ellestad LE (2002) Phytic Acid Chemistry: Influence on Phytin-Phosphorus Availability and Phytase Efficacy. J Appl Poult Res 11: 471-480. 
Citation: Akpoilih BU, Ajani EK, Omitoyin BO (2016) Optimum Dietary Ca/P Ratio and Phytase for Growth and Bone Mineralization in Juvenile Clarias gariepinus Fed Soya Bean-Based Diet. J Aquac Res Development 7: 403. doi:10.4172/2155-9546.1000403

22. Lei XG, Stahl CH (2001) Biotechnological development of effective phytases for mineral nutrition and environmental protection. Appl Microbiol Biotechno 57: $474-481$

23. Singh PK (2008) Significance of phytic acid and supplemental phytase in chicken nutrition: a review. World Poultry Science Journal 64: 553-580.

24. National Research Counçil (NRC) (1993) Nutrient requirements of Fish National Academy Press, Washington, DC.

25. Ballam GC, Nelson TS, Kirby LB (1984) Effect of fibre and phytate source and of calcium and Phosphorus level on phytate hydrolysis in the chick. Poult Sc 63: $333-338$

26. Oberleas D (1973) Phytates In: Toxicants occurring naturally in Foods. National Academic of sciences, Washington DC, USA.

27. Association of Official Analytical Chemists (AOAC) (2006) Official Method of Analysis of the AOAC. Horwitz W (Ed) Eighteenth Edition. Washington DC, USA.

28. Association of official Analytical Chemists (AOAC) (1990) Official Methods of Analysis, 15th edn, Association of Official Analytical Chemists, Washington, DC, USA.

29. BASF (1997) Determination of phytase activity (Relative method). QM System No. E047DA01.

30. Engelen AJ, van der Heeft FC, Randsdorp PHG, Smit ELC (1994) Simple and rapid determination of phytase activity. J AOAC Int 77: 760-764.

31. Cao L, Yang Y, Wang WM, Yakupitiyage A, Yuan DR, Diana JS (2008) Effects of pretreatment with microbial phytase on phosphorous utilization and growth performance of Nile tilapia (Oreochromis niloticus). Aquaculture Nutrition 14: 99-109.

32. Duncan DB (1955) New Multiple Range and Multiple Range F-Tests. Biometrics 11: $1-42$

33. Belal IEH (2005) A review of some fish nutrition methodologies. Bioresource Technology 96: 395-402.

34. Schaefer A, Koppe WM (1995) Effect of a microbial phytase on utilization of native phosphorus by carp in a diet based on soybean meal. Water Sci Technol 31: 149-55.

35. Hughes KP, Soares JH (1998) Efficacy of phytase on phosphorus utilization in practical diets fed to striped bass, Morone saxatilis. Aqua Nutr 4: 133-140.
36. Storebakken T, Shearer KD, Roem AJ (1998) Availability of protein phosphorus and other elements in fishmeal, soy-protein concentrate and phytase-treated soy-protein concentrate-based diets to Atlantic salmon, Salmo salar. Aquaculture. 161: 365-379.

37. Nwanna LC, Kolahsa M, Eisenreich R, Schwarz FJ (2007) Pre-treatment of dietary plant feedstuffs with phytase and its effect on growth and mineral concentration in common carp (Cyprinus carpio L.). Journal of Animal Physiology and Animal Nutrition 92: 677-682.

38. Ravindran V, Cabahug S, Ravindran G, Bryden WL (1999a) Influence of microbial phytase on apparent ileal amino acid digestibility in feedstuffs for broilers. Poult Sci 78: 699-706.

39. Wilson RP, Robinson EH, Gatling DB, Poe WE (1982) Dietary phosphorus requirement of Channel catfish. J Nutr 112: 1197-1202.

40. Lim HS, Namkung H, Um JS, Kang KR, Kim BS, et al. (2000) The effect of phytase supplementation on the performance of broiler chickens fed diets with different levels of non-phytate phosphorus. Asian-Australasian Journal of Animal Sciences 14: 250-257.

41. Nwanna LC, Schwarz FJ (2007) Effect of supplemental phytase on growth phosphorus digestibility and bone mineralization of common carp (Cyprinus carpio L). Aquaculture Research 38: 1037-1044.

42. Denbow DM, Ravindran V, Kornegay ET, YI Z, Hulet RM (1995) Improving phosphorous availability in soyabean meal for broilers by supplemental phytase. Poultry Science 74: 1831-1842.

43. Eyo AA (2003) Fundamentals of fish nutrition and diet development-An overview. National workshop. Fish feed development and feeding practices in Aquaculture. Organized by FISON/NIFER/FAO-NSPFS.

44. Kim JC, Simminsb PH, Mullanc BP, Pluske JR (2005) The effect of wheat phosphorus content and supplemental enzymes on digestibility and growth performance of weaner pigs. Animal Feed Science and Technology 118:139-152.

45. Yan W, Reigh RC (2002) Effects of fungal phytase on utilization of dietary protein and minerals, and dephosphorylation of phytic acid in the alimentary tract of channel catfish Ictalurus punctatus fed an all plant- protein diet. J World Aqua Soc 33: 10-12.

46. Baeverfjord G, Asgard T, Shearer KD (1998) Development and detection of phosphorus deficiency in Atlantic salmon, Salmo salar L., parr and post-smolts. Aquaculture Nutrition 4: 1-11

47. Eeckhout W, de Paepe M (1994) Total phosphorus, phytate-phosphorus and phytase activity in plant feedstuffs. Anim Feed Sci Technol 47: 19-29. 